\title{
Turbulence diffusion effects at material interfaces, with application to the Rayleigh-Taylor instability
}

\author{
Pooya Movahed* and Eric Johnsen ${ }^{\dagger}$ \\ Department of Mechanical Engineering, University of Michigan, Ann Arbor, MI, 48109, US
}

\begin{abstract}
A new set-up is proposed to numerically investigate turbulent multi-material mixing in the presence and in the absence of a gravitational field. The set-up consists of an initial unperturbed interface that separates two fluids in an existing isotropic velocity field. The initial unperturbed interface evolves into a turbulent multi-material mixing region due to the fluctuating velocity field. For simulations without gravity, the initial velocity field decays, while the simulations with gravity are Rayleigh-Taylor unstable, such that the misalignment of the pressure and the density gradients generates baroclinic vorticity feeding the instability. The flow parameters are chosen such that the density fields are different, but that the kinetic energy decays at the same rate in both fluids. Direct numerical simulations are performed using a high-order accurate minimally dissipative kinetic-energy preserving and interface-capturing scheme. Results with and without gravity are compared to investigate flow isotropy and intermittency. The current results suggest that the initial anisotropy in the composition is not sufficient to make the initial isotropic field anisotropic in the absence of gravity.
\end{abstract}

\section{Introduction}

$\mathrm{M}$ ANY astrophysical problems are driven in large part by hydrodynamics. For instance, in core-collapse supernovae, astrophysical jets, stellar formation, etc., large density gradients interact with shock waves or are accelerated, thus leading to hydrodynamic instabilities (e.g., Rayleigh-Taylor, Richtmyer-Meshkov, Kelvin-Helmholtz) that grow and evolve into turbulence. Specifically, the Rayleigh-Taylor instability (RTI), a process by which the misalignment of the pressure, e.g., through a gravitational field, and the density gradients at unstably stratified interfaces generates baroclinic vorticity, can transition from a laminar flow field to a fully mixed turbulent field. ${ }^{1}$ In many cases, the pressure gradient is produced by a hydrostatic gravitational field.

A better understanding of turbulent mixing can significantly improve our knowledge of late-time behavior of these instabilities. Turbulent mixing is a multiscale process in which fluids are entrained at the largest scales, transported (dispersed) by eddies of varying sizes, and molecularly mixed at the smallest scales where diffusion acts. ${ }^{2}$ This multiscale process is an effective means to mix different fluids with several consequences in nature and engineering. While turbulent mixing is beneficial in combustion and supernova explosion, it can provide challenges in the design of inertial confinement fusion capsules as mixing between the fuel and ablator quenches the fuel, preventing high pressures and temperatures to be achieved. Turbulent mixing is categorized based on its influence on flow dynamics into three different levels based on Dimotakis: ${ }^{2}$

- Level I: Flow dynamics are decoupled from mixing. Examples include mixing of density-matched gases, advection of passive scalars, e.g., the dispersion of nonreacting trace markers, such as pollutants.

- Level II: Mixing and flow dynamics are coupled. For instance, mixing in the RTI has a direct consequence on flow dynamics as it can significantly change the amount of baroclinic vorticity deposited in the mixing region.

${ }^{*}$ Graduate Student, Department of Mechanical Engineering, 1231 Beal Ave., 2043 Walter E. Lay Automotive Laboratory, Ann Arbor, MI 48109-2133, AIAA Member.

${ }^{\dagger}$ Assistant Professor, Department of Mechanical Engineering, 1231 Beal Ave., 2043 Walter E. Lay Automotive Laboratory, Ann Arbor, MI 48109-2133, AIAA Member. 
- Level III: Mixing is coupled to the dynamics by modifying the fluid(s). The changes can be in composition, density, pressure, or enthalpy, e.g., due to chemical reactions in combustion.

Level II mixing, the focus of this work, has been mostly studied in the context of the multi-mode RTI. ${ }^{3,2,4}$ Theoretical and experimental investigations of such flows are challenging due to the highly nonlinear dynamics and wide range of spatial and temporal scales. Numerical simulation has emerged as a useful tool that can provide a wealth of data otherwise difficult to obtain. Over the past few decades, computations of these instabilities have been able to test the accuracy of assumptions in analytical models and address some of the fundamental questions regarding these hydrodynamic instability-driven turbulent fields. ${ }^{4,5}$ However, there remain significant unknowns in these flows, particularly regarding the late-time turbulent multi-material mixing behavior, especially with multi-mode initial conditions. Previous direct numerical simulations (DNS) suggest that while these flows become anisotropic at large scales, they remain isotropic at small scales. ${ }^{4,3}$ In the RTI, the anisotropy at large scales is typically related to the directional gravitational force as well as the initial anisotropy in the mass fraction and density profiles. Much of the efforts in past studies was focused on predicting the growth rate; flow intermittency was only reported recently. ${ }^{6,7}$

In this paper, we propose a novel set-up to investigate Level II mixing in the presence and in the absence of gravity. Two fluids in an existing decaying isotropic field are considered. The initial unperturbed interface between the two fluids evolves into a turbulent multi-material mixing region. The simulations without gravity allows us to determine whether anisotropy in the composition alone leads to anisotropy in the velocity field. Flow isotropy and intermittency in different directions are investigated in detail. This paper is organized as follows. In Section II, the governing equations and the numerical method are described briefly. The decaying isotropic turbulence problem is discussed in section III. Sections IV and V contain the description and results for the new set-up with and without gravity, respectively.

\section{Problem framework}

\section{A. Physical model}

The focus of the present work is on mixing mechanisms between two perfect gases due to a turbulent field in the presence and in the absence of gravity. The non-dimensional compressible Navier-Stokes equations are considered:

$$
\begin{aligned}
& \frac{\partial \rho}{\partial t}+\frac{\partial}{\partial x_{j}}\left(\rho u_{j}\right)=0, \\
& \frac{\partial\left(\rho u_{i}\right)}{\partial t}+\frac{\partial}{\partial x_{j}}\left(\rho u_{i} u_{j}+p \delta_{i j}\right)=\frac{1}{R e} \frac{\partial \tau_{i j}}{\partial x_{j}}+\rho g_{i}, \\
& \frac{\partial E}{\partial t}+\frac{\partial}{\partial x_{j}}\left[u_{j}(E+p)\right]=\frac{1}{R e}\left[\frac{\partial}{\partial x_{j}}\left(u_{i} \tau_{i j}\right)+\frac{\partial}{\partial x_{j}}\left[k \frac{\partial T}{\partial x_{j}}\right]+\frac{1}{S c} \frac{\partial}{\partial x_{j}}\left[\rho \frac{\partial Y}{\partial x_{j}}\right]\left(h_{1}-h_{2}\right)\right]+\rho u_{j} g_{j}, \\
& \frac{\partial \rho Y}{\partial t}+\frac{\partial \rho u_{j} Y}{\partial x_{j}}=\frac{1}{R e S c} \frac{\partial}{\partial x_{j}}\left[\rho \frac{\partial Y}{\partial x_{j}}\right],
\end{aligned}
$$

where $\rho$ is the density, $p$ the pressure, $u_{i}$ the velocity, $g_{i}$ the gravity, $E$ the total energy per unit volume, $T$ the temperature, $Y$ the mass fraction, $k$ the thermal conductivity, $h$ the enthalpy, Re the scaled Reynolds number, and $S c$ is the Schmidt number. The viscous stress tensor $\tau_{i j}$ for Newtonian fluids is given by

$$
\tau_{i j}=\mu\left[\left(\frac{\partial u_{i}}{\partial x_{j}}+\frac{\partial u_{j}}{\partial x_{i}}\right)-\frac{2}{3} \frac{\partial u_{k}}{\partial x_{k}} \delta_{i j}\right]
$$

where $\mu$ is the viscosity. The viscosity (and similarly for the thermal conductivity) of the mixture is determined from Herning and Zipper ${ }^{8}$ approximation:

$$
\mu=\frac{\mu_{1} Y_{1} M_{1}^{-1 / 2}+\mu_{2} Y_{2} M_{2}^{-1 / 2}}{\mu_{1} M_{1}^{-1 / 2}+\mu_{2} M_{2}^{-1 / 2}},
$$

where $M$ is the molecular weight. The ideal gas law for a binary mixture reads

$$
\frac{p}{\rho}=\frac{\bar{R} T}{M}, \quad \text { with } \quad \frac{1}{M}=\frac{Y_{1}}{M_{1}}+\frac{Y_{2}}{M_{2}}
$$


where $\bar{R}$ is the universal gas constant.

The two fluids can have different density, pressure, temperature, molecular weight, viscosity, and thermal conductivity based on the initial set-up. Non-dimensionalization is performed using the light-fluid density, $\rho_{1}$, the length $l=L /(2 \pi)$ where $L$ is the computational domain width, the velocity $u_{r e f}=\sqrt{g l}$, the pressure $p=\rho_{1} u_{r e f}^{2}$, the light-fluid gas constant, $R_{1}$, and the temperature of unity. The specific heats ratio, $\gamma$, is set to 1.4 for both fluids; the Schmidt number is unity corresponding to a gas-gas mixture; the Prandtl number, $\mathrm{Pr}$, is set to 0.7 in both fluids and the gravity vector is $(0,0,-1)$ in the non-dimensional space corresponding to the Froude number, $F r$, of unity for the simulations with gravity. The non-dimensional quantities are related to the scaled variables as

$$
R e=\frac{\rho_{1} l u_{r e f}}{\mu_{1}}, \quad S c=\frac{\mu_{1}}{\rho_{1} D}, \quad \operatorname{Pr}=\frac{c_{p} \mu}{k}, \quad F r=\frac{u_{r e f}}{\sqrt{g l}},
$$

where $D$ is the mass diffusivity, and the specific heat at constant pressure $c_{p}$ can be written:

$$
c_{p}=\frac{\gamma}{\gamma-1} R
$$

For simplicity, we set $\mu_{1}=1$ and define the scaled Reynolds number, Re, in Eq. 12 later.

\section{B. Numerical method}

Finite differences are used to numerically solve the compressible Navier-Stokes equations for multiple fluids. ${ }^{9}$ A sixth-order explicit central scheme is used to approximate advection fluxes. Aliasing errors are minimized using the split form for advection. In particular, the cubic advection terms in Eq. 1 are expanded as

$$
\frac{\partial}{\partial x_{j}}\left(\rho u_{j} \phi\right)=\frac{1}{2} \frac{\partial}{\partial x_{j}}\left(\rho u_{j} \phi\right)+\frac{1}{2} u_{j} \frac{\partial}{\partial x_{j}}(\rho \phi)+\frac{1}{2} \rho \phi \frac{\partial u_{j}}{\partial x_{j}},
$$

following Blaisdell et al. ${ }^{10}$ where $\phi=\left(1, u_{i},(E+p) / \rho, Y_{i}\right)$. The flux of Ducros et al. ${ }^{11}$ for the split form is used to satisfy the summation by part in periodic domains and ensures conservation as well. Diffusive terms are discretized in non-conservative form:

$$
\frac{\partial}{\partial x}\left(\mu \frac{\partial u}{\partial x}\right)=\mu \frac{\partial^{2} u}{\partial x^{2}}+\frac{\partial \mu}{\partial x} \frac{\partial u}{\partial x}
$$

thus resulting in better accuracy, robustness, spectral representation of diffusive effects at high wavenumbers, and preventing odd-even decoupling. ${ }^{12}$ A third-order accurate strong stability preserving (SSP) RungeKutta scheme is used for explicit time marching. ${ }^{13}$ When needed, nonreflecting boundary conditions are implemented in characteristic form using one-sided differences. ${ }^{14}$ The code is written in FORTRAN and parallelized using MPI, including HDF5 for parallel I/O.

\section{Freely decaying homogeneous isotropic turbulence}

In this section, we discuss the decay of homogeneous isotropic turbulence, ${ }^{15}$ which is used to initialize the problems under consideration. The initial conditions consist of a random solenoidal velocity field in a triple periodic box of size $[2 \pi]^{3}$ with an energy spectrum that satisfies

$$
E(k)=16 \sqrt{\frac{2}{\pi}} \frac{u_{r m s}^{2}}{k_{0}} \frac{k^{4}}{k_{0}^{4}} \exp \left(\frac{-2 k^{2}}{k_{0}^{2}}\right) \sim k^{4} \exp \left(\frac{-2 k^{2}}{k_{0}^{2}}\right),
$$

where $k_{0}$ is the most energetic wavenumber and $\lambda_{0}=2 / k_{0}$ is the initial Taylor microscale. ${ }^{15,16}$ The density and pressure fields are initially uniform. The turbulent Mach number, $M_{t}$, and Reynolds number based on the Taylor microscale, $R e_{\lambda}$, are the main parameters defined as

$$
M_{t}=\frac{\sqrt{\left\langle u_{i} u_{i}\right\rangle}}{\langle c\rangle}, \quad R e_{\lambda}=\frac{\langle\rho\rangle u_{r m s} \lambda}{\langle\mu\rangle}
$$


where

$$
u_{r m s}=\sqrt{\frac{\left\langle u_{i} u_{i}\right\rangle}{3}}, \quad \lambda^{2}=\frac{\left\langle u_{i}^{2}\right\rangle}{\left\langle\left(\frac{\partial u_{i}}{\partial x_{i}}\right)^{2}\right\rangle} .
$$

Here $c$ is the sound speed, and $\lambda$ is the Taylor microscale. The important time scale of the problem is the eddy turn-over time defined as $\tau=\lambda_{0} / u_{r m s_{0}}$. The viscosity, $\mu$, is set to unity and the scaled Reynolds number can be obtained in terms of the initial Taylor-scale Reynolds number:

$$
R e=R e_{\lambda}\left[\frac{\rho u_{r m s, 0} \lambda_{0}}{\mu}\right]^{-1}
$$

For each $k_{0}$, the velocity field is generated on the finest grid $\left(N^{3}=512^{3}\right)$ and filtered spectrally onto coarser grids. The initial energy spectrum corresponds to Batchelor turbulence $\left(E(k) \sim k^{4}\right)$ at low wavenumbers and has an exponential decay at high wavenumbers in the viscous subrange. The energy cascade transfers energy to higher modes during the process, resulting an initial increase in the vorticity field and in an inertial range. During this transition, different turbulent modes (acoustic, vorticity, and entropy) also reach an equilibrium state. ${ }^{17}$ Fig. 1 shows the evolution of different quantities of interest at $R e_{\lambda}=60-200$ and $M_{t}=100$. The skewness of the velocity derivatives,

$$
S=\frac{\left\langle\frac{1}{3}\left(\frac{\partial u_{i}}{\partial x_{i}}\right)^{3}\right\rangle}{\left\langle\frac{1}{3}\left(\frac{\partial u_{i}}{\partial x_{i}}\right)^{2}\right\rangle^{1.5}}
$$

reaches a maximum negative value of approximately -0.55 at $t \approx 0.6 \tau$, consistent with past work. ${ }^{18} \mathrm{~A}$ skewness of zero corresponds to a Gaussian field, expected for an isotropic problem; however, the skewness of the velocity derivatives becomes negative as a direct consequence of the energy cascade. The vorticity reaches its peak after an initial transient. This transient and the maximum peak increases as the initial $R e_{\lambda}$ is increased. Another observation is that $\lambda$ decreases as the initial $R e_{\lambda}$ increases, as expected since larger $R e_{\lambda}$ leads to a greater scale separation. Since there is no external source providing energy to the turbulence, the total kinetic energy decays due to viscous dissipation. This results in a continuous decay of $M_{t}$ as well.

Mach number effects are summarized in Fig. 2 where several simulations at $R e_{\lambda}=100$, and $M_{t}=0.1-0.5$ are reported. Shocklets may form at sufficiently high $M_{t}$ and may thus require shock capturing, but the central scheme in the split form used here was found stable for all of the simulations. Although the average values of normalized dilatation at $M_{t}=0.1$ is negligible, it increases at higher $M_{t}$ as compressibility effects become more important.

\section{Multi-material mixing in the absence of gravity}

\section{A. Initial set-up}

The computational domain consists of a rectangular parallelepiped with square cross-section of size $L \times L \times$ $10 L$, with $L=2 \pi$ (see Fig. 3). The initial mass fraction field is generated in $z$ without any perturbations in the $x-y$ plane (Fig. 4):

$$
Y(z)=\frac{1}{2}\left[1-\operatorname{erf}\left(\frac{z-z_{0}}{\delta}\right)\right]
$$

where $z_{0}=0$ is the mid-plane location separating the two fluids corresponding to $Y_{1}=0.5$. The $\delta=\frac{8}{128} L$ corresponds to the sharpest interface profile that the central scheme is capable of resolving with no shockcapturing on a $N=128$ point per $L$ grid. The density in the light fluid, $\rho_{1}$, is the reference density. Pressure is uniform initially in the absence of gravity. To achieve an isothermal field the properties of the heavy and light fluids are related as follows:

$$
\frac{R_{2}}{R_{1}}=\frac{M_{1}}{M_{2}}=\frac{\rho_{1}}{\rho_{2}}, \quad p_{\text {initial }}=T_{\text {initial }}=2 \pi L\left(\rho_{1}+\rho_{2}\right) .
$$




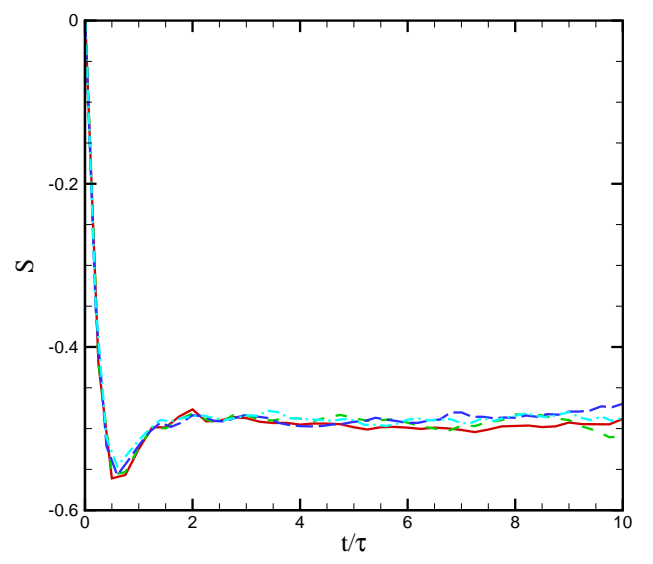

(a) Skewness.

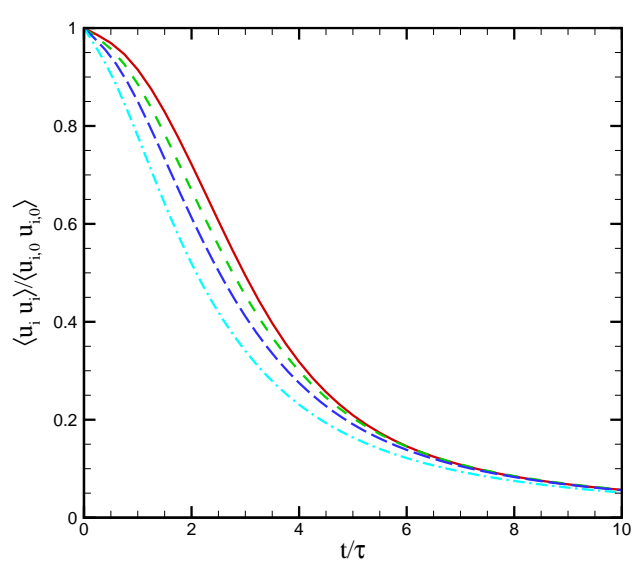

(c) Normalized kinetic energy.

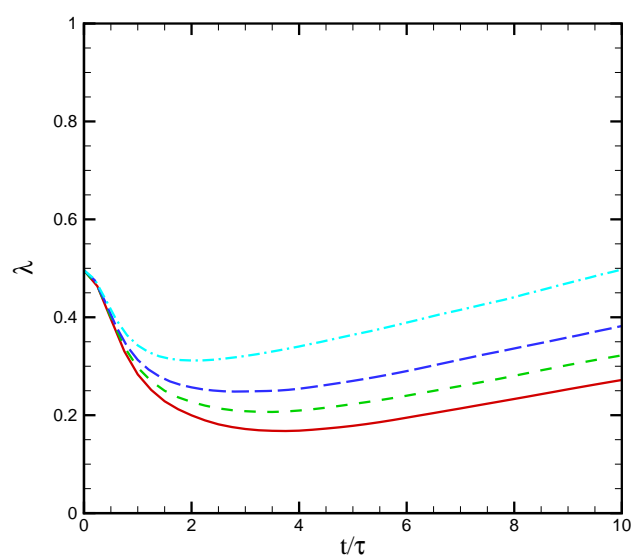

(e) Taylor microscale.

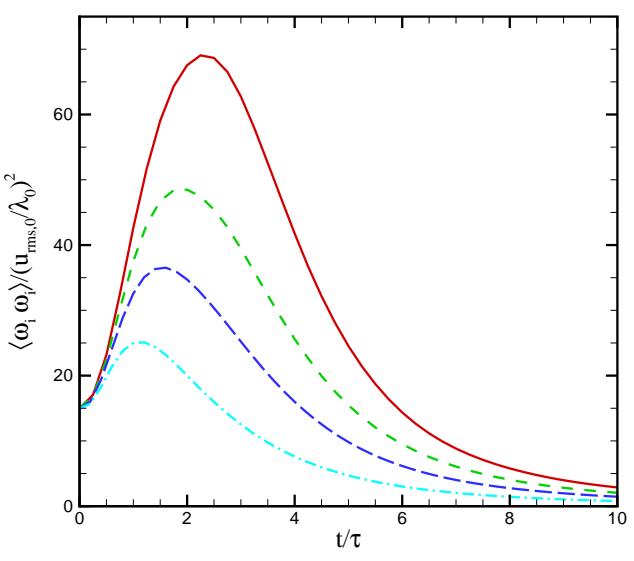

(b) Enstrophy.

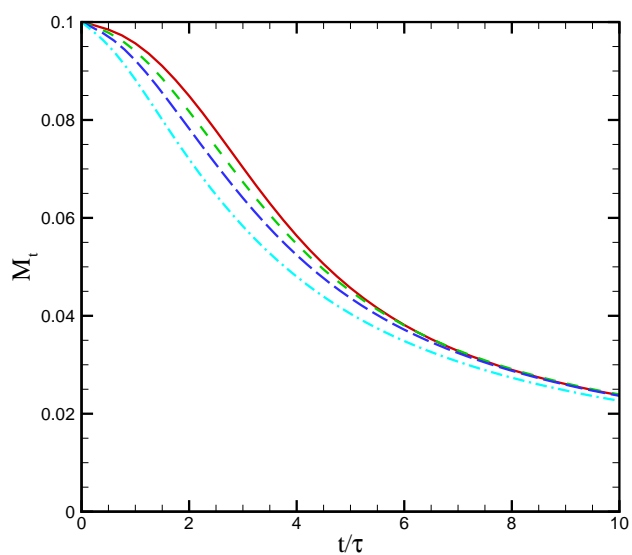

(d) Turbulent Mach number.

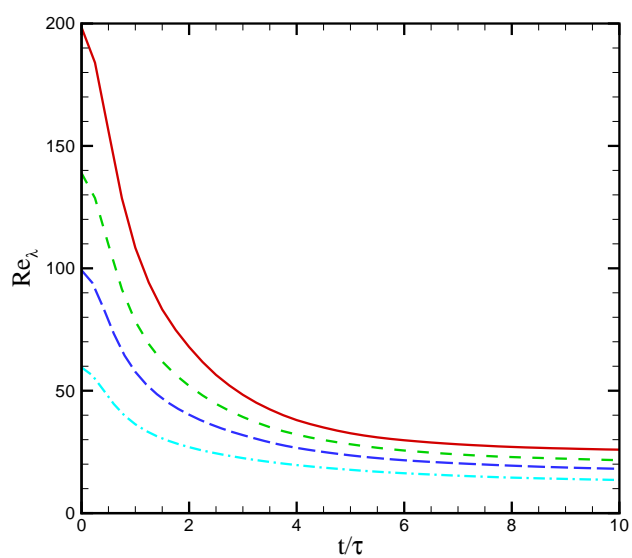

(f) $R e_{\lambda}$.

Figure 1. Temporal evolution of different quantities for the decaying isotropic turbulence problem at $R e_{\lambda}=60$ (gray), 100 (blue), 140 (green), 200 (red) and $M_{t}=0.1$.

In the mixing region, the initial density profile is obtained by assuming the limit of two miscible incompressible flows as: ${ }^{3}$

$$
\frac{1}{\rho}=\frac{Y_{1}}{\rho_{1}}+\frac{Y_{2}}{\rho_{2}} .
$$




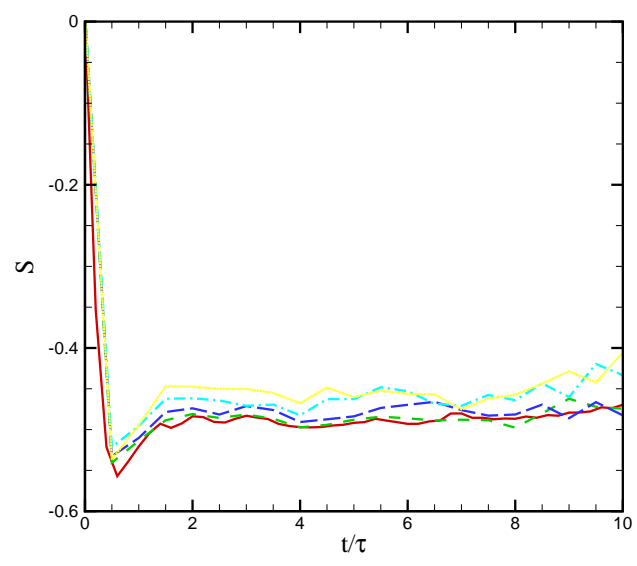

(a) Skewness.

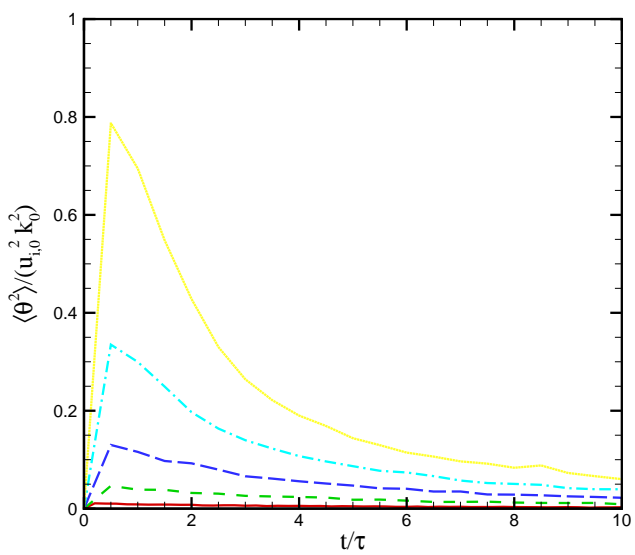

(c) Dilatation.

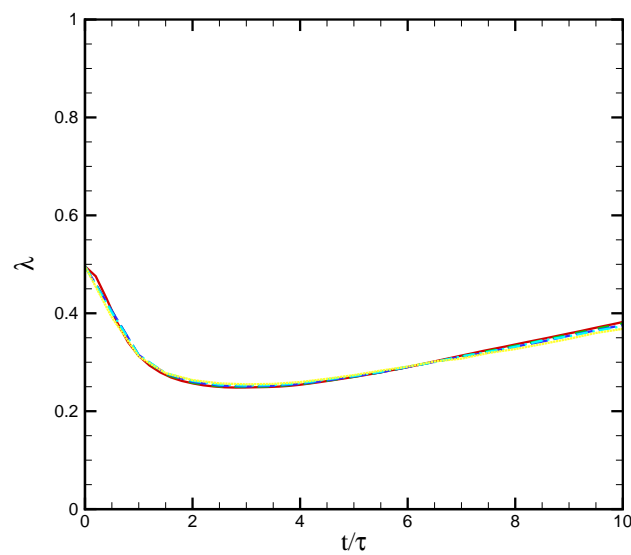

(e) Taylor microscale.

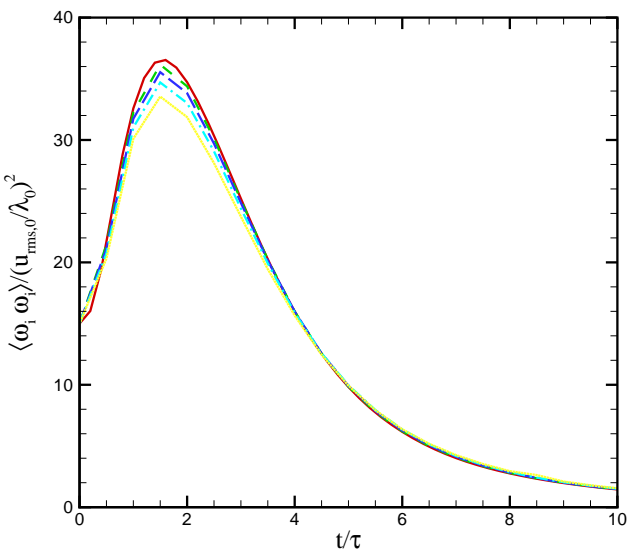

(b) Enstrophy.

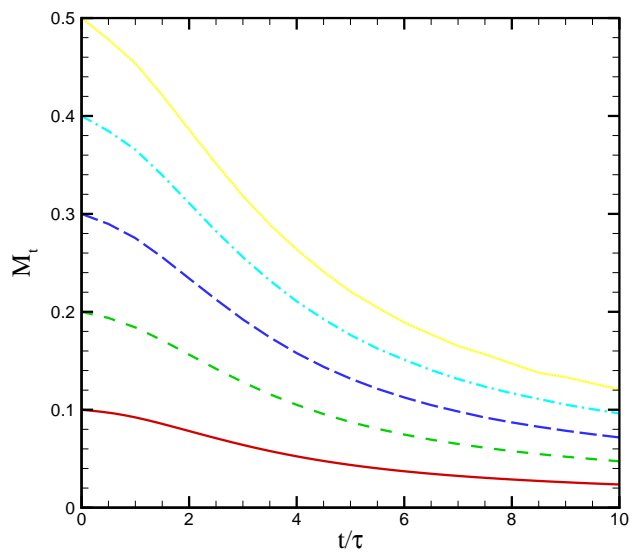

(d) Turbulent Mach number.

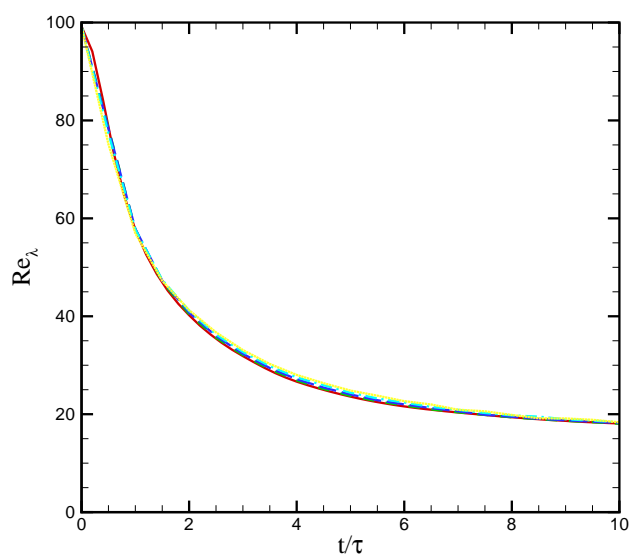

(f) $R e_{\lambda}$.

Figure 2. Temporal evolution of different quantities for the decaying isotropic turbulence problem at $R e_{\lambda}=100$ and $M_{t}=0.1$ (red), 0.2 (green), 0.3 (blue), 0.4 (gray), 0.5 (yellow).

The velocity field described in section III is used to initialize the problem. An initial velocity field is generated in a box of size $L^{3}$ and, given the periodicity of the problem, ten boxes are put next to each other in the $z$-direction to make up the full domain. Although Thompson's approach ${ }^{14}$ is followed to simulate 


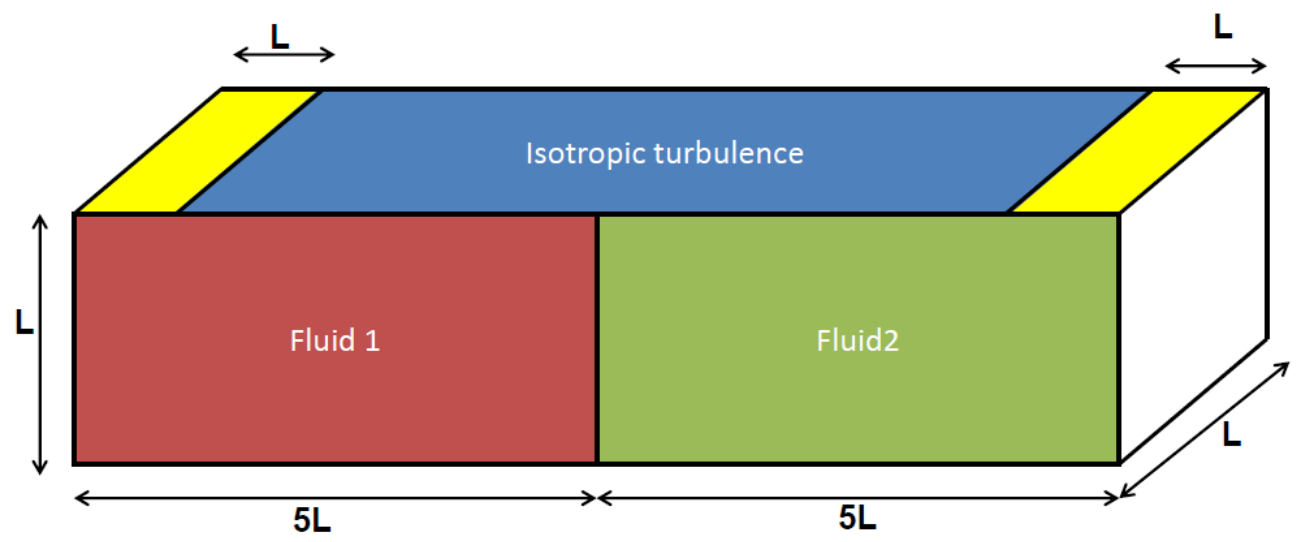

Figure 3. Computational set-up for the mixing problem. An unperturbed diffuse material interface separating two fluids evolves to a multi-material mixing region in the presence of an existing isotropic turbulent field.

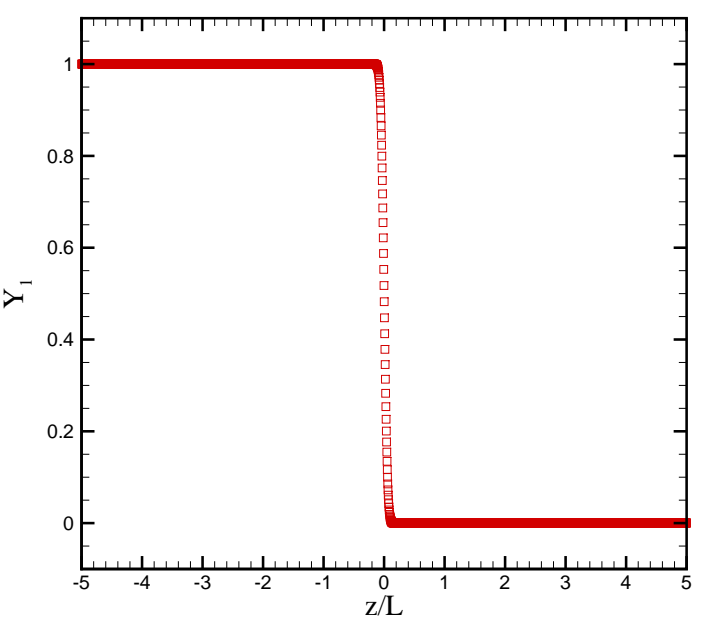

(a) Mass fraction.

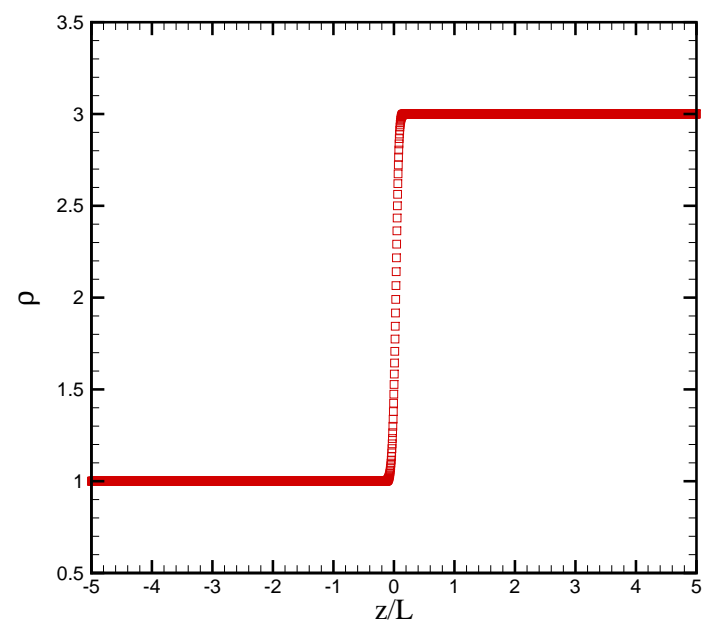

(b) Density.

Figure 4. Initial mass fraction (left) and density (right) profiles for $\rho_{2} / \rho_{1}=3$, and $N=256$ per $L$.

non-reflecting boundary conditions in the $z$-direction at the ends, numerical errors may occur if the initial turbulent region is extended up to the boundaries. To avoid these challenges, an error function is used to damp the turbulence close to the boundaries and a long domain length in the $z$-direction $(10 L)$ is considered to minimize the effects of boundaries on the evolution of the mixing region. To prevent generating unphysical waves at the interface in the presence of finite mass physical diffusion, an additional velocity is prescribed at the interface:

$$
u_{i}=-\frac{D}{R e S c} \frac{1}{\rho} \frac{\partial \rho}{\partial x_{i}} .
$$

In the current set-up, pressure is initially uniform. Thus, unlike in the Rayleigh-Taylor instability, no significant baroclinic vorticity is generated at the interface (other than from the interaction between acoustic and entropy modes). Thus, the turbulence decays similarly to that described in section III, as there is no source to feed energy into the system. While the initial velocity field is isotropic and homogeneous, an inhomogeneity is introduced into the set-up at the interface due to the composition and density change across the interface in the $z$-direction. Mixing between the two fluids can be considered as simplified Level II mixing. The key turbulent properties in the heavy and light fluids are related as follows:

$$
\frac{R e_{\lambda 2}}{R e_{\lambda 1}}=\frac{\rho_{2} \mu_{1}}{\rho_{1} \mu_{2}}, \quad M_{t 2}=M_{t 1} \sqrt{\frac{\rho_{2}}{\rho_{1}}} .
$$




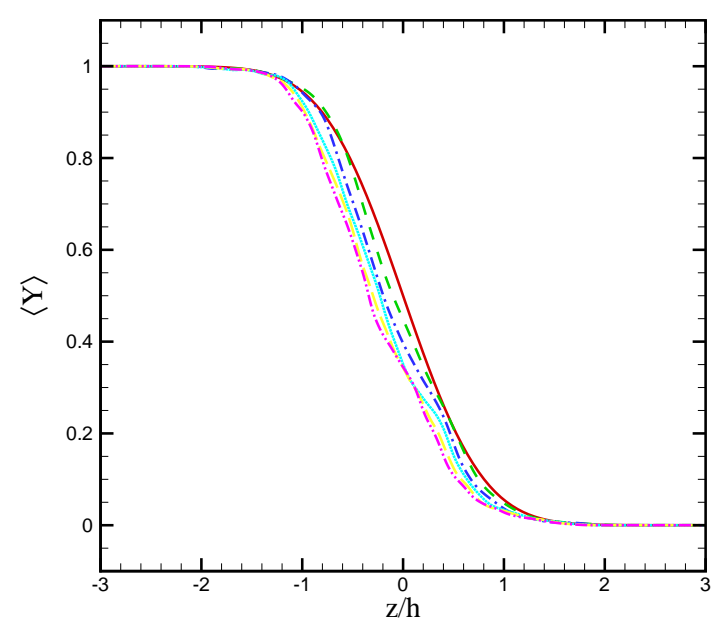

(a) $0-5 \tau$.

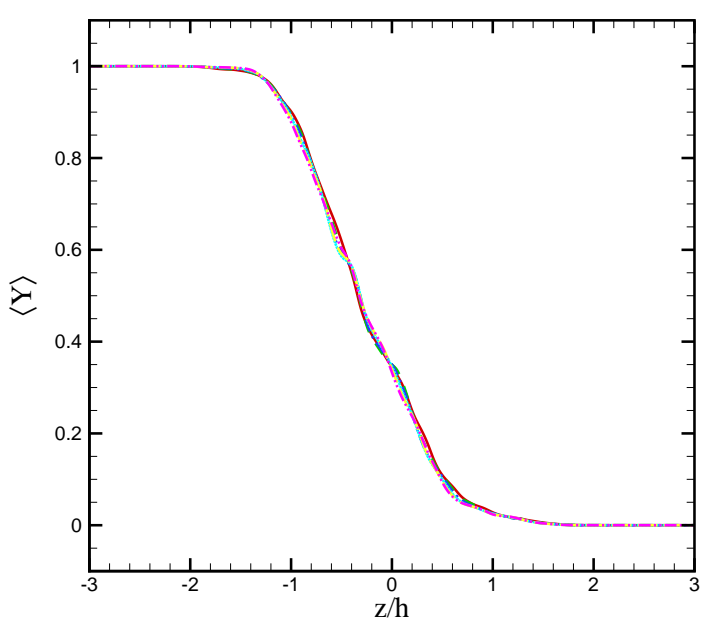

(b) $5-20 \tau$.

Figure 5. Temporal evolution of the mass fraction field.

\section{B. Results}

In this section, we will consider the following initial conditions to investigate Level II mixing and quantify isotropy and intermittency in the mixing region:

$$
\frac{\rho_{2}}{\rho_{1}}=\frac{\mu_{2}}{\mu_{1}}=3, \quad R e_{\lambda_{1}}=R e_{\lambda_{2}}=60, \quad M_{t, 1}=0.1, \quad S c=1
$$

\section{Growth rate}

The average velocity in all directions is zero for the initial velocity field, i.e., there is no mean advection. Thus, any variation in the mass fraction is a consequence of the flow being transported by turbulence (eddies of different sizes) in the presence of finite mass diffusion. The mixing between the two fluids falls in the category of Level II mixing as the two fluids have different densities, and the interdiffusion of the two fluids into each other introduces an additional energy transfer mechanism through the following term in the energy equation:

$$
\frac{1}{R e S c} \frac{\partial}{\partial x_{j}}\left[\rho \frac{\partial Y}{\partial x_{j}}\right]\left(h_{1}-h_{2}\right) .
$$

It is noted that Soret and Dufour diffusion processes are expected to be small and are thus neglected.

The initial fluctuating velocity field perturbs the interface and spikes/bubbles of the heavier/lighter fluid penetrate into the lighter/heavier fluid. The spike and bubble locations are defined as the position where $\left\langle Y_{1}\right\rangle \leq 0.99$ and $\left\langle Y_{1}\right\rangle \geq 0.01$, respectively, where the brackets $\langle\cdot\rangle$ represent the mean average operator in the $x-y$ plane. The amplitude of growth is defined as the average of the bubble and spike growth; this amplitude is thus sensitive to the threshold chosen. To minimize statistical fluctuations, amplitude can also be measured as follows: ${ }^{3}$

$$
h=2 \int_{-\infty}^{\infty} \min \left(\left\langle Y_{1}\right\rangle,\left\langle Y_{2}\right\rangle\right) d z
$$

Fig. 6 shows the time evolution of the bubble, spike and amplitude. The Atwood number $A=\left(\rho_{2}-\right.$ $\left.\rho_{1}\right) /\left(\rho_{2}+\rho_{1}\right)$ is 0.5 . For simulations with gravity at this Atwood number, the spike growth rate is expected to be larger than that of the bubble. A similar behavior is observed here in the absence of gravity. The higher growth rate of the spike can be associated with the higher mass-average kinetic energy of the heavier fluid, which makes it easier for the heavier fluid to push the lighter fluid and penetrate into it. The mass fraction field shown in Fig. 5 suggests that the mixing region grows self-similarly after $5 \tau$. One-dimensional turbulence-diffusion models can be used to describe the observed growth rate. ${ }^{19}$ 


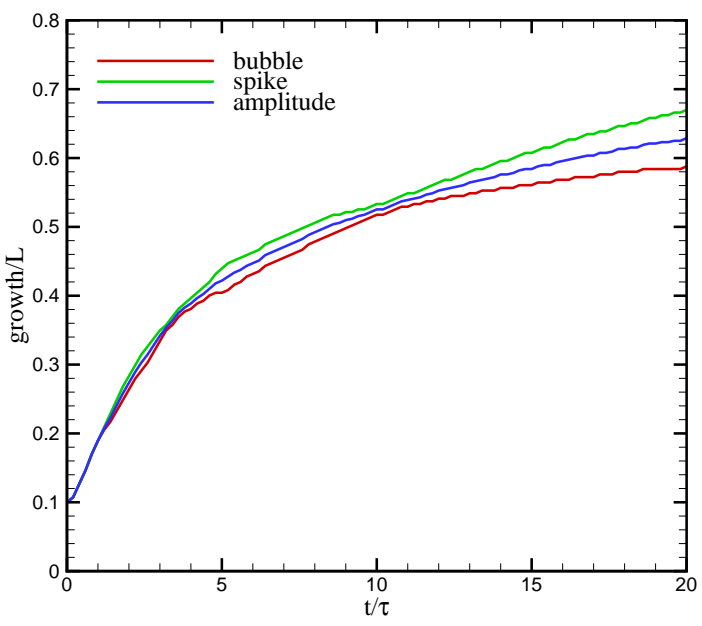

(a) Growth.

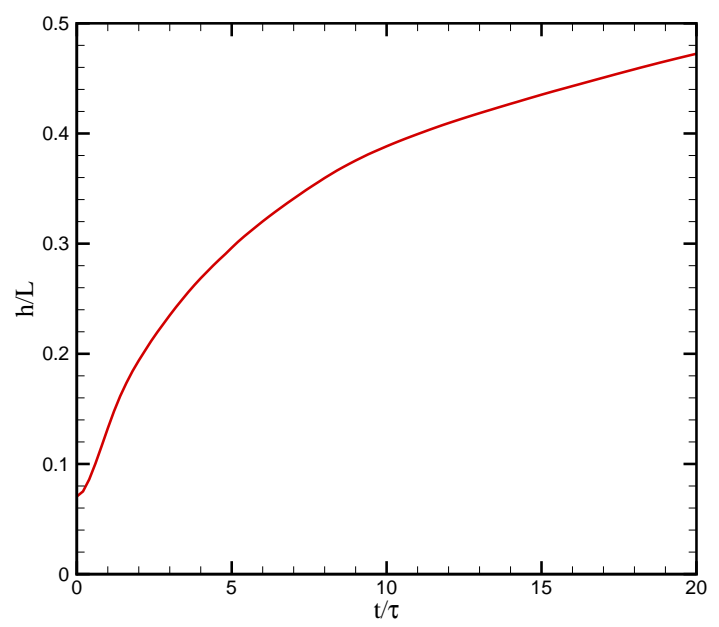

(b) Entrainment length.

Figure 6. Temporal evolution of the amplitude of the mixing region.

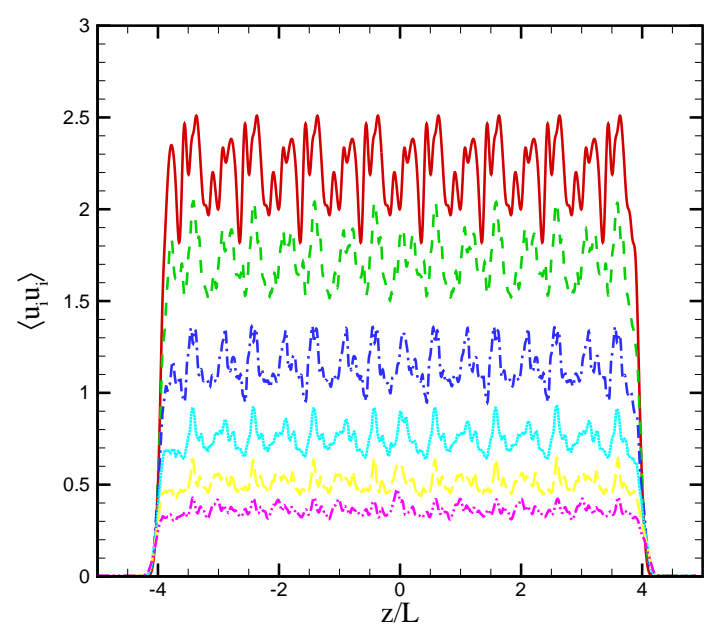

(a) Kinetic energy.

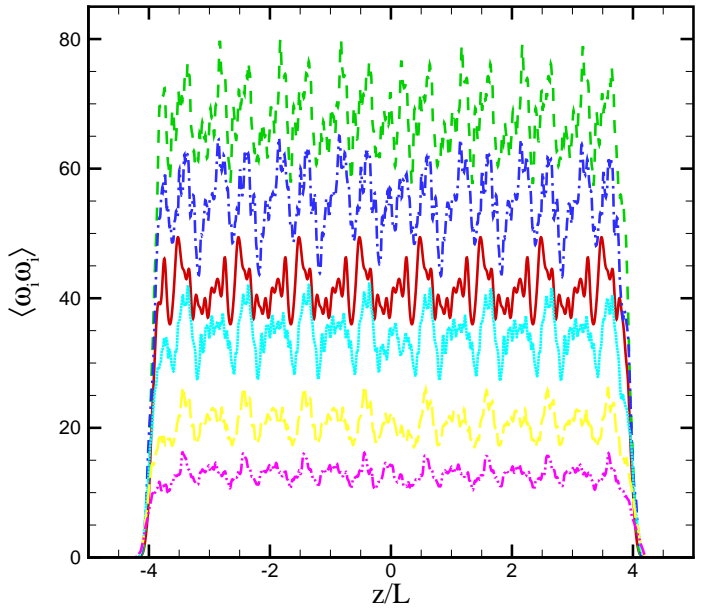

(b) Enstrophy.

Figure 7. Temporal evolution of (a) kinetic energy and (b) enstrophy during the first five eddy-turn over time.

\section{Turbulence statistics}

The spatial distribution of the kinetic energy, $\left\langle u_{i} u_{i}\right\rangle$, and enstrophy, $\left\langle\omega_{i} \omega_{i}\right\rangle$ are depicted at different times in Fig. 7. Although the two fluids have different densities, the velocity field decays at the same rate in both fluids since the kinematic viscosity, and thus $R e_{\lambda}$, are the same in the two fluids. This feature is desirable here as an energy jump across the interface leads to flow anisotropy. ${ }^{20,21}$ The same decay rate ensures that any anisotropy at later times, if observed, is a consequence of the anisotropy in the composition rather than the energy jump across the interface.

Fig. 8 shows the root-mean-square (rms) fluctuations of the density and mass fraction, and also the velocity-mass fraction correlation. At each $x-y$ plane, the rms of a fluctuating field $X$ is defined as:

$$
X_{r m s}=\left\langle(X-\langle X\rangle)^{2}\right\rangle \text {. }
$$

The fluctuation profiles at the spike front are very similar when $h$ is used for non-dimensionalization. This is in agreement with the self-similar growth described in the previous section. The fluctuations also decay as 
the velocity field decays due to viscosity. There is a positive scalar flux from fluid 1 to fluid 2 . The results suggest that the center of high intensity in the mixing region is skewed toward the lighter fluid. This shift of the highest intensity fluctuations from the mid-plane is in agreement with the higher growth observed for the spike compared to the bubble.

Next, we consider the different length scales relevant to the problem. These length scales are evaluated in the mixing region, defined as the region between $x-y$ planes of mean mass fraction values of 0.25 and 0.75. The Taylor microscale can be considered representative of the internal viscous shear-layer thickness associated with large-scale motions spanning the full transverse extent of the flow, i.e., the smallest scale of the large-scale eddy motions. ${ }^{2}$ The Kolmogorov scale is the smallest scale of turbulence associated with viscous dissipation of turbulent kinetic energy. The Taylor microscale, $\lambda_{i}$, and an estimate of the Kolmogorov microscale, $\eta_{i}$, in the $i$ th direction can be defined as: ${ }^{4}$

$$
\lambda_{i}(z, t)=\left[\frac{\left\langle u_{i}^{2}\right\rangle}{\left\langle\left(\partial u_{i} / \partial x_{i}\right)^{2}\right\rangle}\right]^{1 / 2}, \quad \eta_{i}(z, t)=\left(\frac{(\nu R e)^{3}}{\epsilon_{i}}\right)^{1 / 4}(\text { no sum in } i),
$$

where

$$
\epsilon_{i}(z, t)=15 \nu \operatorname{Re}\left\langle\left(\frac{\partial u_{i}}{\partial x_{i}}\right)^{2}\right\rangle(\text { no sum in } i)
$$

is the directional dissipation rate. These scales can be used to quantify the isotropy of a turbulent field at different scales in different directions. Fig. 9 suggests that the turbulence remains isotropic at both the Taylor and the Kolmogorov microscales, despite the anisotropy in the composition and density at the interface.

\section{Intermittency of velocity derivatives}

The small-scale intermittency of the velocity field is measured by looking at the directional skewness $(S)$ and kurtosis $(K)$ of the velocity derivatives. These quantities are defined as:

$$
S_{\frac{\partial u_{i}}{\partial x_{j}}}=\frac{\left\langle\left(\frac{\partial u_{i}}{\partial x_{j}}\right)^{3}\right\rangle}{\left\langle\left(\frac{\partial u_{i}}{\partial x_{j}}\right)^{2}\right\rangle^{1.5}}, \quad K_{\frac{\partial u_{i}}{\partial x_{j}}}=\frac{\left\langle\left(\frac{\partial u_{i}}{\partial x_{j}}\right)^{4}\right\rangle}{\left\langle\left(\frac{\partial u_{i}}{\partial x_{j}}\right)^{2}\right\rangle^{2}} .
$$

A comparison between the flow intermittency of our set-up and the decaying isotropic turbulence problem can also be used as a means to measure flow isotropy. Fig. 10 summarizes the skewness and kurtosis of the $z$-velocity derivatives in different directions. Kurtosis remains relatively close to four on average. Skewness remains close to either 0 or -0.5 on average. These values correspond to an isotropic field as reported in section III. This suggests that the level of anisotropy is small for the current set-up in the absence of gravity. We will compare these statistics in the next section with a similar set-up in the presence of gravity to quantify the effects of gravity on small-scale intermittency and isotropy.

Since we are considering compressible fluids, it is of interest to investigate the skewness and mass fraction of the mass-averaged momentum derivatives as well. These quantities are similarly defined as

$$
S_{\frac{\partial \rho u_{i}}{\partial x_{j}}}=\frac{\left\langle\left(\frac{\partial \rho u_{i}}{\partial x_{j}}\right)^{3}\right\rangle}{\left\langle\left(\frac{\partial \rho u_{i}}{\partial x_{j}}\right)^{2}\right\rangle^{1.5}}, \quad K_{\frac{\partial \rho u_{i}}{\partial x_{j}}}=\frac{\left\langle\left(\frac{\partial \rho u_{i}}{\partial x_{j}}\right)^{4}\right\rangle}{\left\langle\left(\frac{\partial \rho u_{i}}{\partial x_{j}}\right)^{2}\right\rangle^{2}} .
$$

Fig. 11 shows that higher levels of intermittency in the mass-averaged velocity derivatives exist in the $z$ -

direction. In other directions, the skewness and kurtosis remain similar to those in Fig. 10 since the density variation in each $x-y$ plane is not very large.

\section{Rayleigh-Taylor instability}

\section{A. Initial set-up}

Numerical simulations of the Rayleigh-Taylor instability are traditionally initialized by perturbing the interface either through density perturbations or by converting these density perturbations to velocity perturba- 


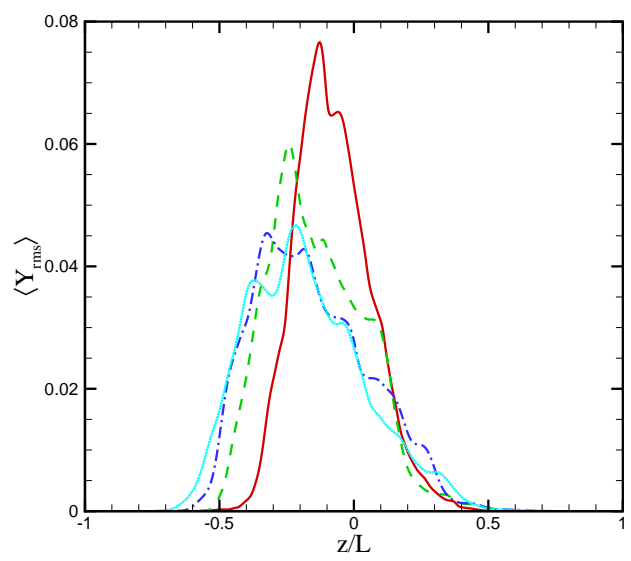

(a) $\left\langle Y_{r m s}\right\rangle$.

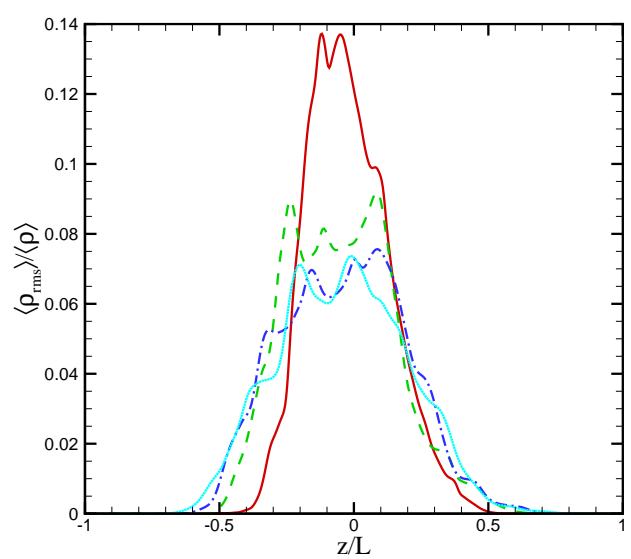

(c) $\left\langle\rho_{r m s}\right\rangle /\langle\rho\rangle$.

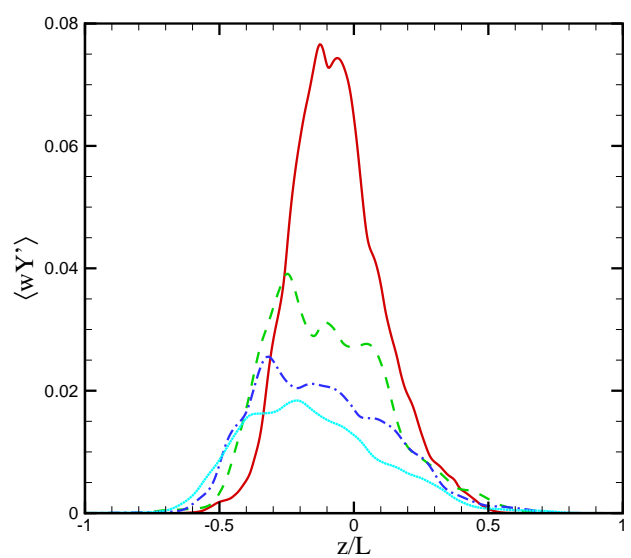

(e) $\left\langle w Y^{\prime}\right\rangle$.

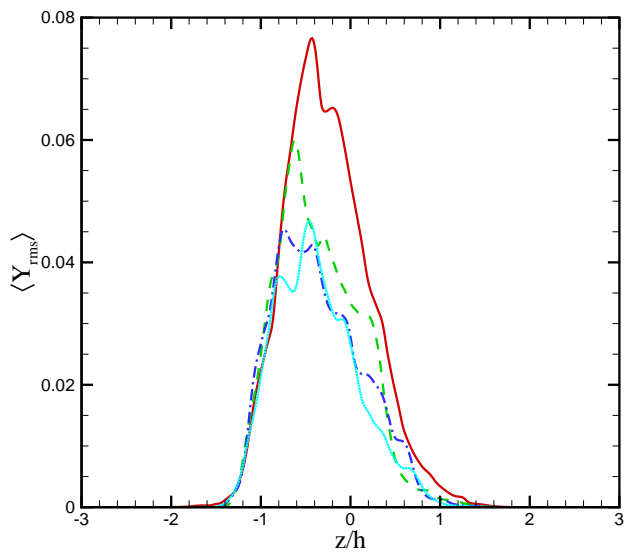

(b) $\left\langle Y_{r m s}\right\rangle$.

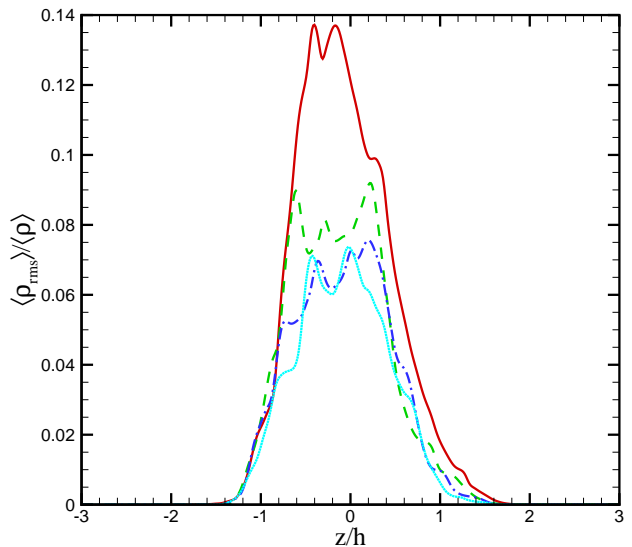

(d) $\left\langle\rho_{r m s}\right\rangle /\langle\rho\rangle$.

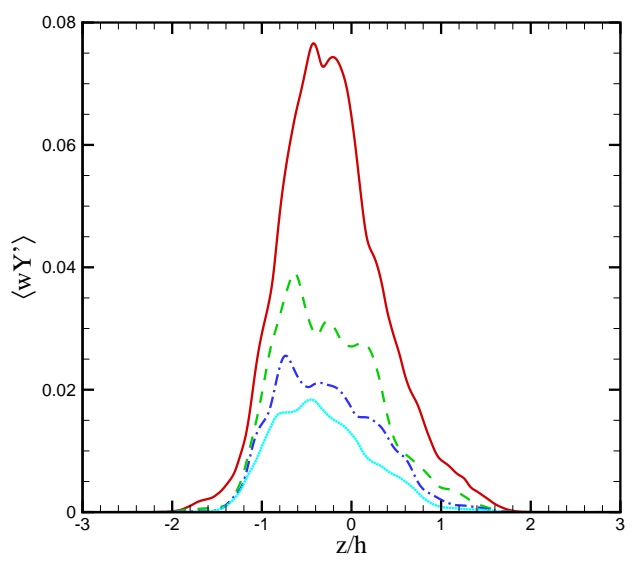

(f) $\left\langle w Y^{\prime}\right\rangle$.

Figure 8. Spatial distribution of different turbulence statistics at $5 \tau$ (red), $10 \tau$ (green), 15 $\tau$ (blue), and $20 \tau$ (gray).

tions using linear theory. ${ }^{6}$ In this study, the same set-up as that described in Section A is used, the only difference being an acceleration field in the negative $z$-direction. Thus, the turbulent velocity field perturbs the interface. 


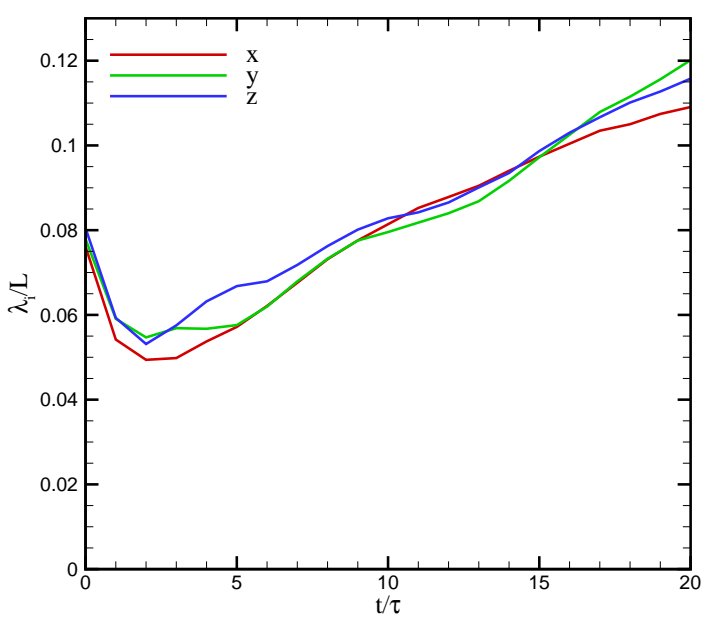

(a) Taylor microscale.

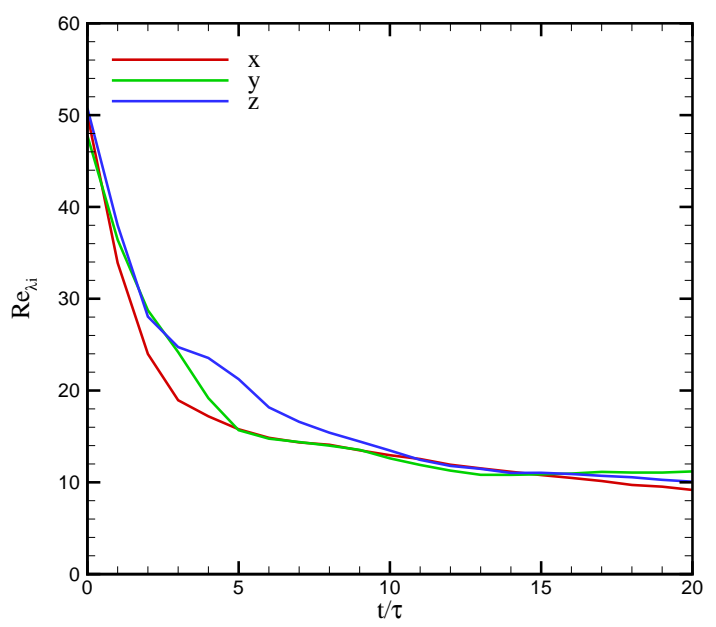

(b) $R e_{\lambda_{i}}$.

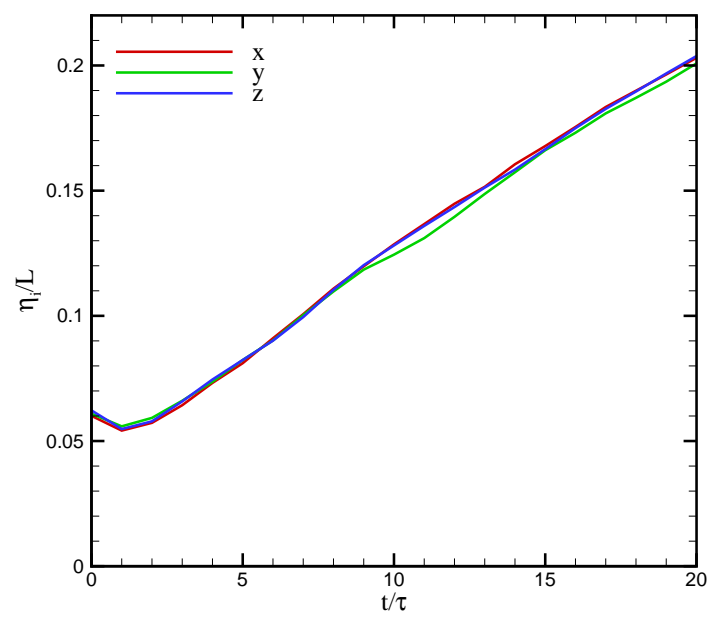

(c) Kolmogorov scale.

Figure 9. Temporal evolution of different quantities.

The initial mass fraction and density fields are the same as the set-up discussed in Section A. The following equations are combined to obtain the pressure and temperature in both fluids: ${ }^{22,23}$

$$
R=R_{0} \sum_{i=1}^{2} \frac{Y_{i}}{M_{i}}, \quad p=\rho R T, \quad \frac{d p}{d z}=-\rho g .
$$

Here both temperature and pressure vary in each fluid. The computational domain again consists of a rectangular parallelepiped with square cross-section of size $L \times L \times 10 L$, with $L=2 \pi$. The density in the light fluid, $\rho_{1}$, is the reference density and $\rho_{2}=3 \rho_{1}$ corresponding to an Atwood number of $A=\frac{\rho_{2}-\rho_{1}}{\rho_{2}+\rho_{1}}=0.5$. To achieve a similar dissipation rate for the initial decaying turbulence (i.e., same $R e_{\lambda}$ ), the properties of the heavy and light fluids are related as follows:

$$
\frac{R_{2}}{R_{1}}=\frac{M_{1}}{M_{2}}=\frac{\mu_{1}}{\mu_{2}}=\frac{\rho_{1}}{\rho_{2}}, \quad R e_{\lambda 1}=R e_{\lambda 2}, \quad M_{t 2}=M_{t 1} \sqrt{\frac{\rho_{2}}{\rho_{1}}} .
$$




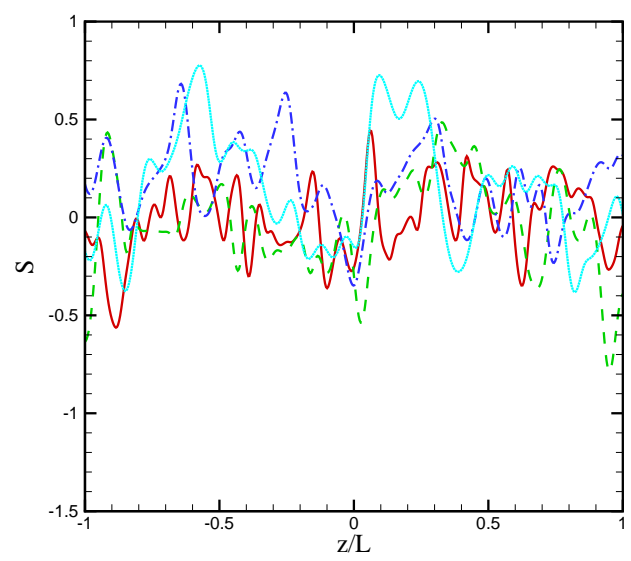

(a) $S_{\partial w / \partial x}$.

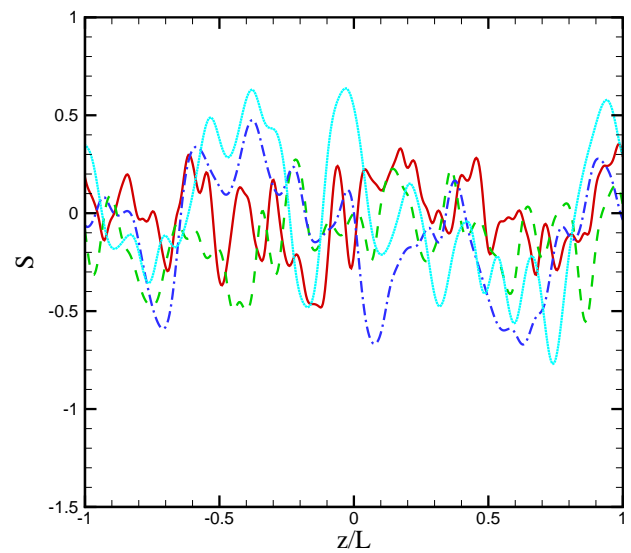

(c) $S_{\partial w / \partial y}$.

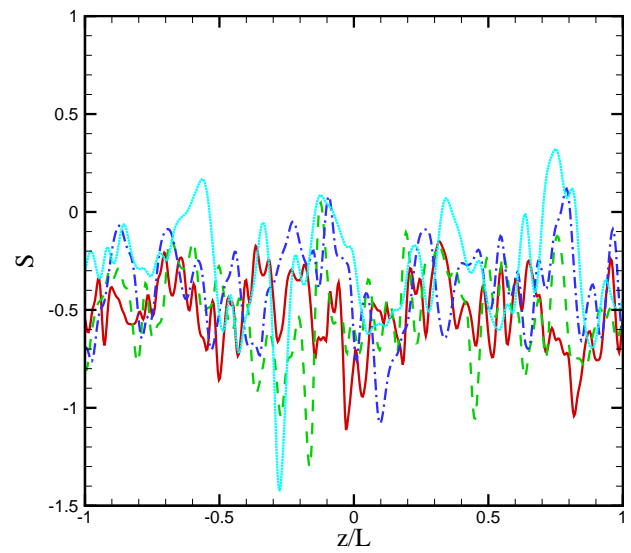

(e) $S_{\partial w / \partial z}$.

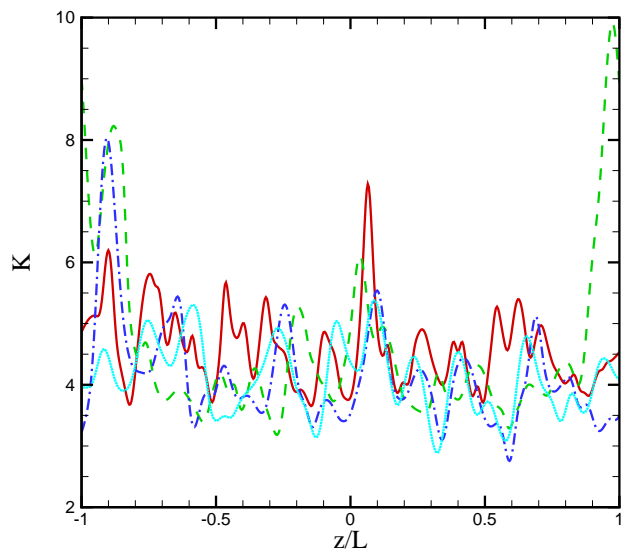

(b) $K_{\partial w / \partial x}$.

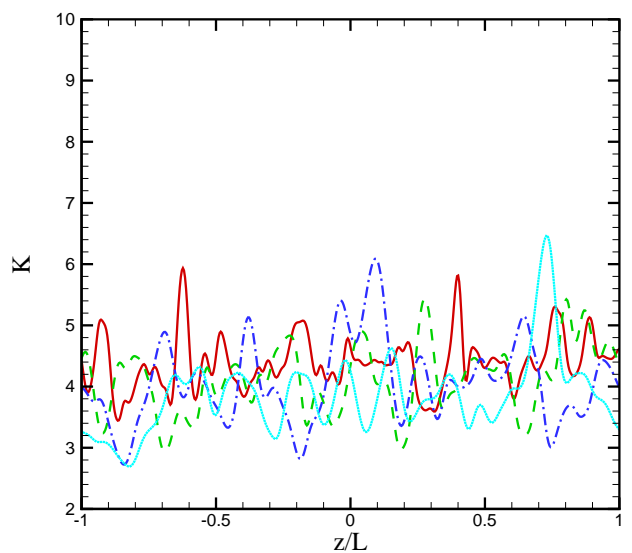

(d) $K_{\partial w / \partial y}$.

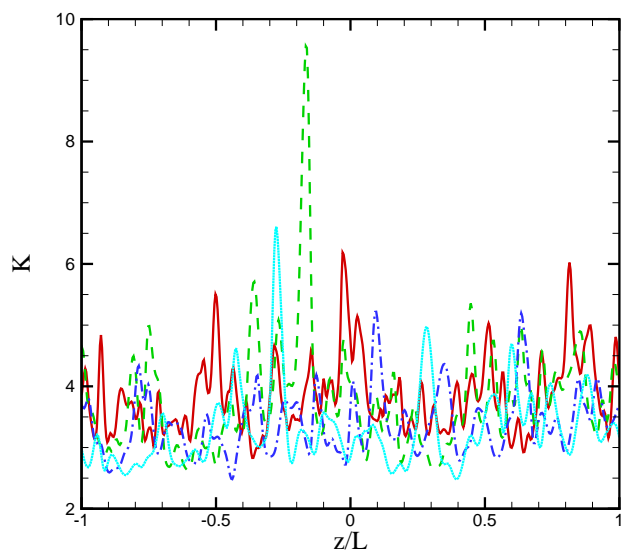

(f) $K_{\partial w / \partial z}$.

Figure 10. Spatial distribution of skewness and kurtosis at $5 \tau$ (red), $10 \tau$ (green), $15 \tau$ (blue), and $20 \tau$ (gray).

\section{B. Results}

Here, we report the results for two sets of RTI simulations with the new set-up at a resolution of $N=256$ points per box width until $20 \tau$ for $k_{0}=4$, and $80 \tau$ for $k_{0}=16$. Note that $\tau$ for $k_{0}=4$ is four times larger 


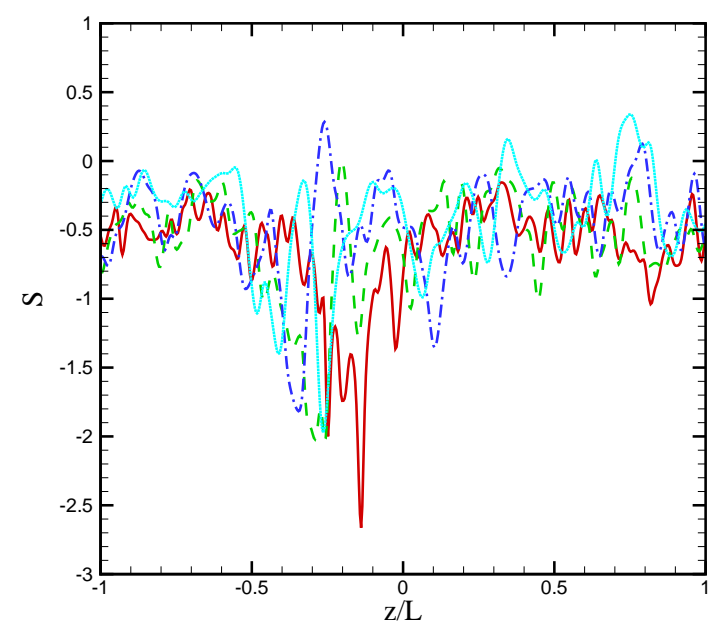

(a) Mass-averaged skewness.

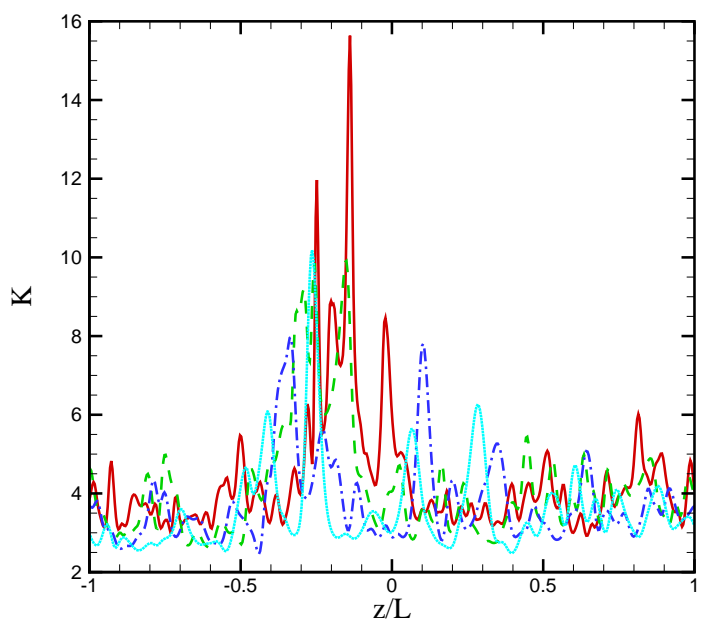

(b) Mass-averaged Kurtosis.

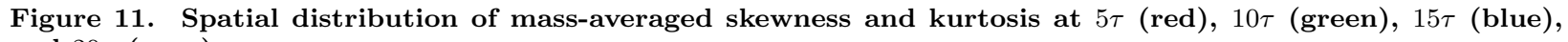
and $20 \tau$ (gray).

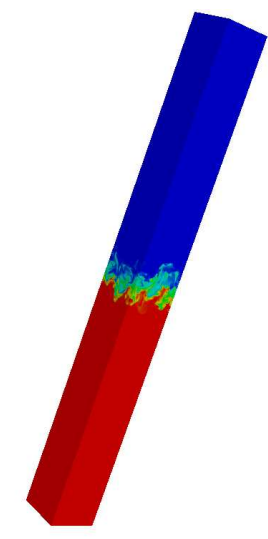

(a) Full domain.

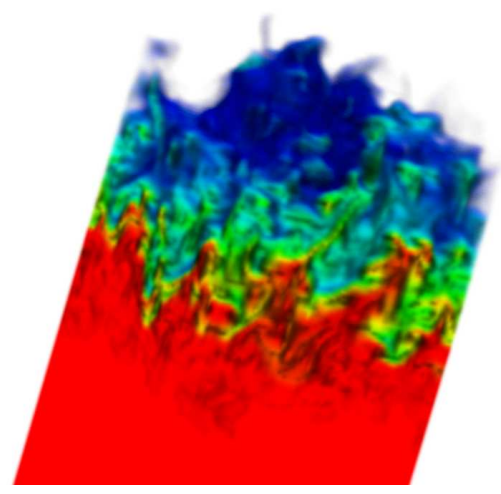

(b) Zoomed in.

Figure 12. Mass fraction contours from the Rayleigh-Taylor set-up for $k_{0}=4$, and $t / \tau=5$.

than $\tau$ for $k_{0}=16$, such that simulations are completed at the same time in terms of the RTI development. Figs. 12 and 13 show contours of the mixing region after $5 \tau$. The initial fluctuating velocity field perturbs the interface, and the baroclinic vorticity provides energy for the growth of the instability. As observed in the mass fraction field, spikes/bubbles of the heavier/lighter fluid penetrate into the lighter/heavier fluid. Fig. 14 shows the time evolution of the bubble and spike. The initial growth rate is dominated by turbulence diffusion in the early stages. As the initial Taylor microscale is larger for $k_{0}=4$, a higher growth is observed for this case during the initial transient, up to $t \approx 5 \tau$; turbulence diffusion is the main mechanism for the growth of the mixing region. The spike growth is higher than the bubble growth, as expected for $A=0.5$ after this initial transient period.

Fig. 15 shows the temporal evolution of the Taylor microscale and the Kolmogorov microscale for $k_{0}=4,16$. Unlike the simulations with no gravity where the Kolmogorov microscale keeps increasing, the Kolmogorov microscale for the RT unstable set-up remains almost uniform after the initial transient. At this time, a balance develops between the energy generated by the instability and the amount of energy that is dissipated at small-scales due to viscosity. ${ }^{24}$ Results also demonstrate a clear anisotropy in the Taylor microscale in the $z$-direction. Results with $k_{0}=16$ result in a value of $R e_{\lambda}$ in the $z$-direction beyond the 


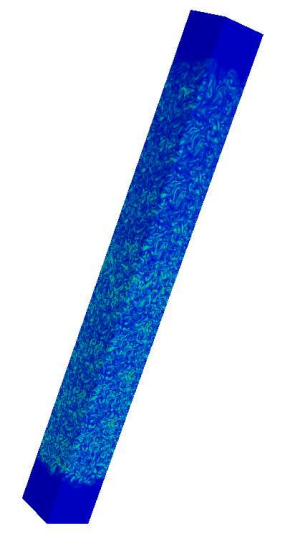

(a) Full domain.

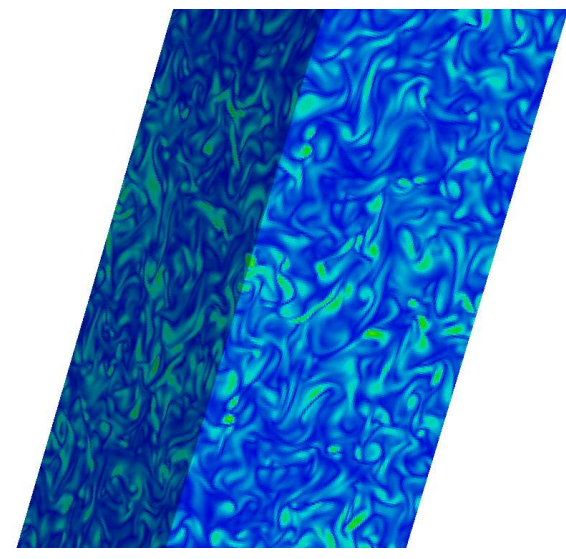

(b) Zoomed in.

Figure 13. Vorticity contours from the Rayleigh-Taylor set-up for $k_{0}=4$, and $t / \tau=5$.

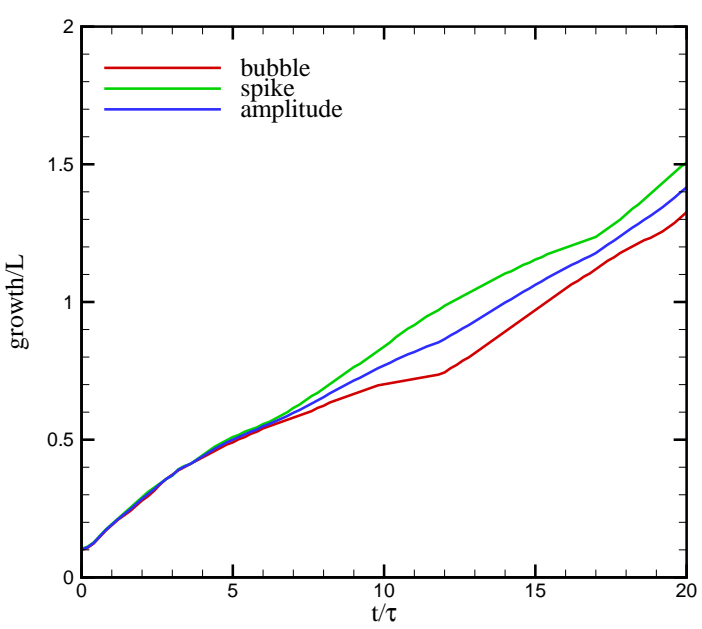

(a) $k_{0}=4$.

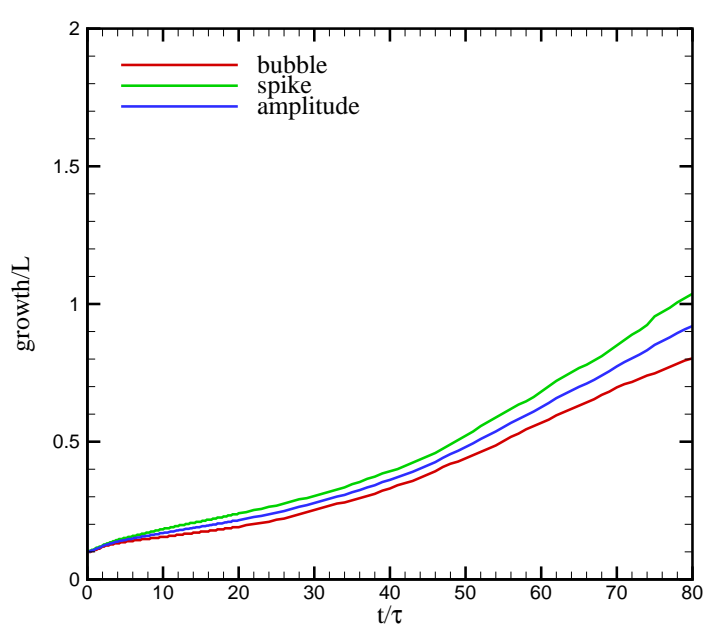

(b) $k_{0}=16$.

Figure 14. Temporal evolution of the mixing region width for $k_{0}=4$ (left) and $k_{0}=16$ (right).

mixing transition. ${ }^{2}$ The flow remains almost isotropic in the $x$ - and $y$-directions. However, in the $z$-direction, it is isotropic at the Kolmogorov scale as well, although the large scales and Taylor microscale are not.

Fig. 16 shows the skewness and kurtosis for the $z$-velocity derivatives in different directions. With increasing time, the results starts to deviate more and more from the results with no gravity. Higher values of kurtosis and skewness are achieved. In particular, high levels of intermittency are achieved at the bubble/spike fronts. These peaks start to move toward the boundaries as the mixing region grows. 


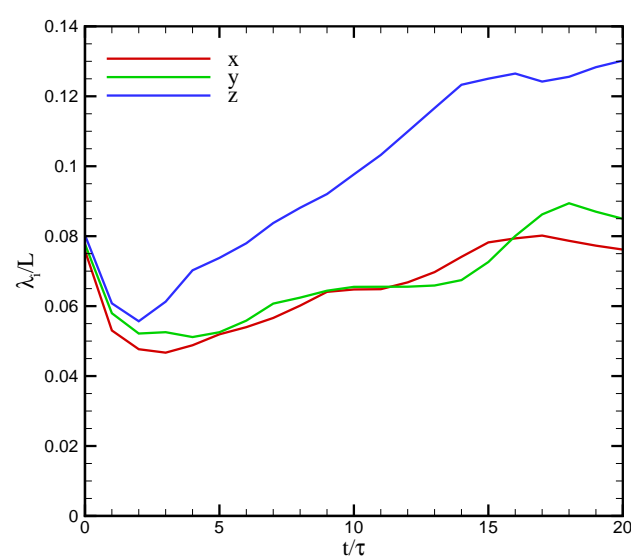

(a) Taylor microscale.

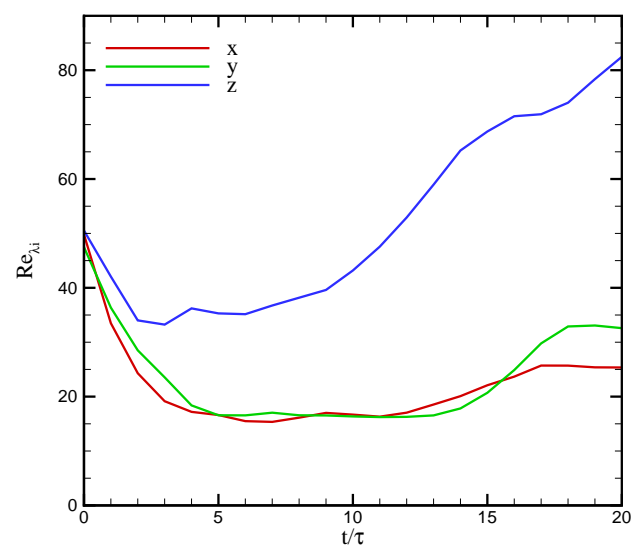

(c) $R e_{\lambda}$.

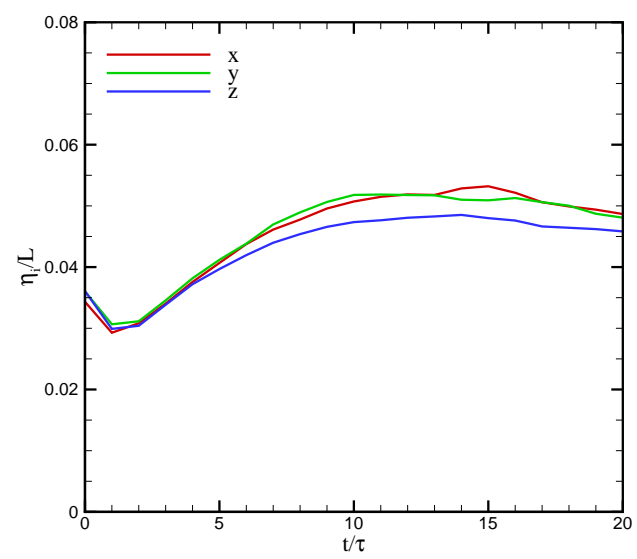

(e) Kolmogorov microscale.

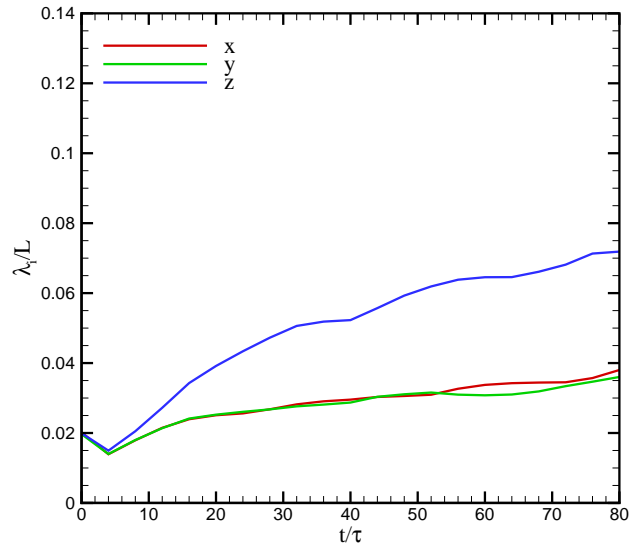

(b) Taylor microscale.

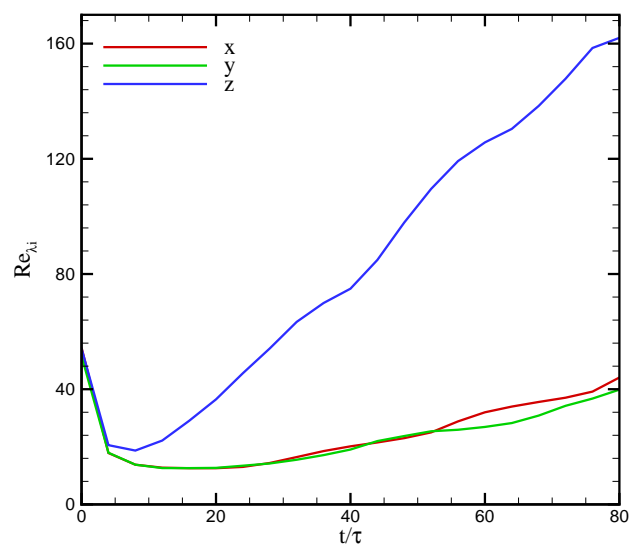

(d) $R e_{\lambda}$

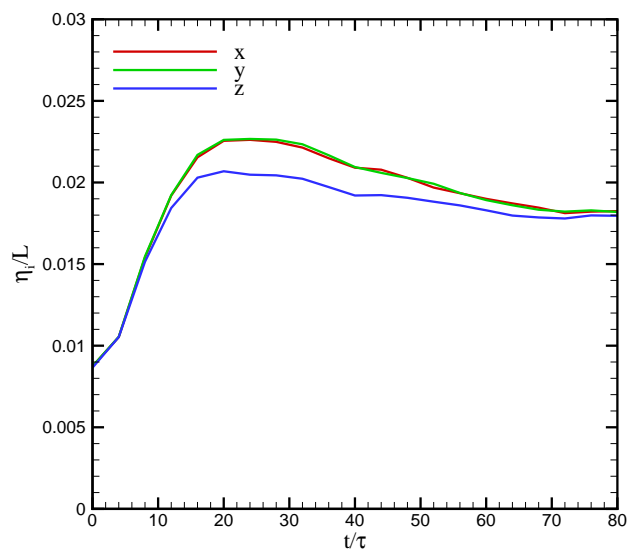

(f) Kolmogorov microscale.

Figure 15. Temporal evolution of different quantities at $k_{0}=4$ (left) and $k_{0}=16$ (right). 


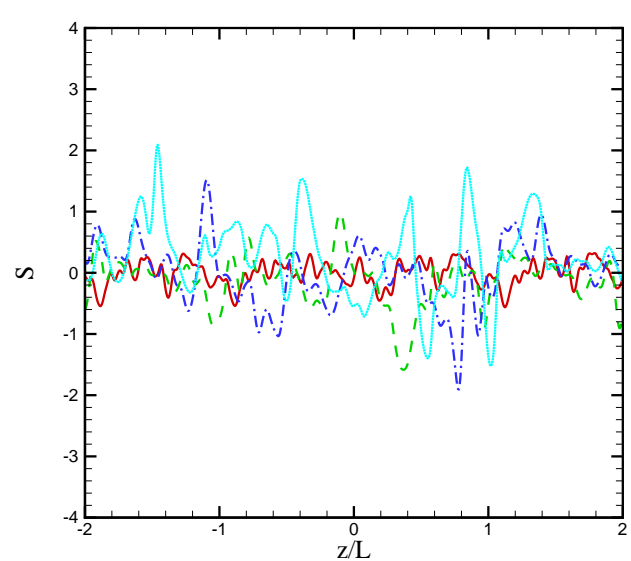

(a) $S_{\partial w / \partial x}$.

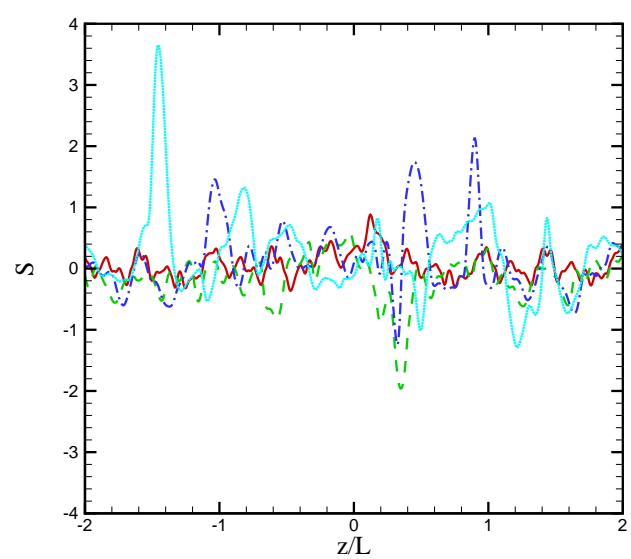

(c) $S_{\partial w / \partial y}$.

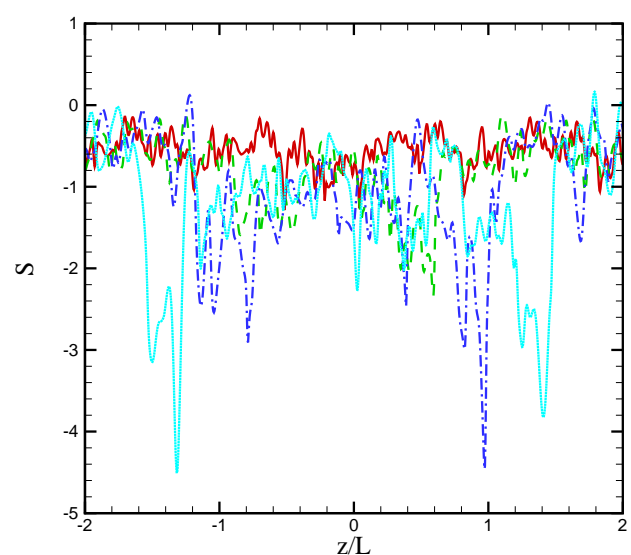

(e) $S_{\partial w / \partial z}$.

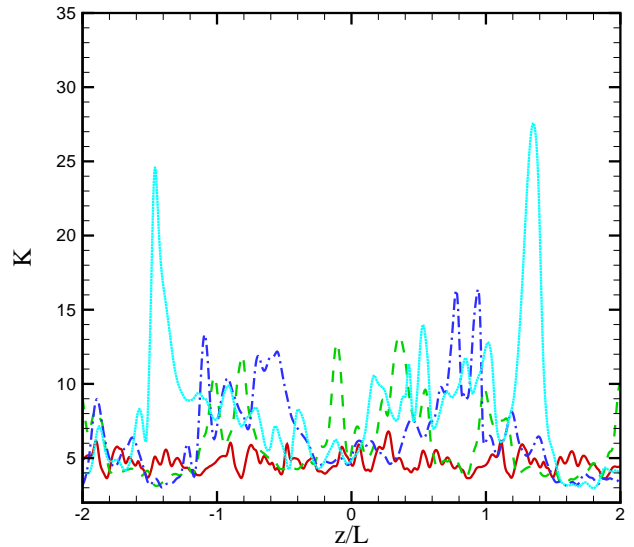

(b) $K_{\partial w / \partial x}$.

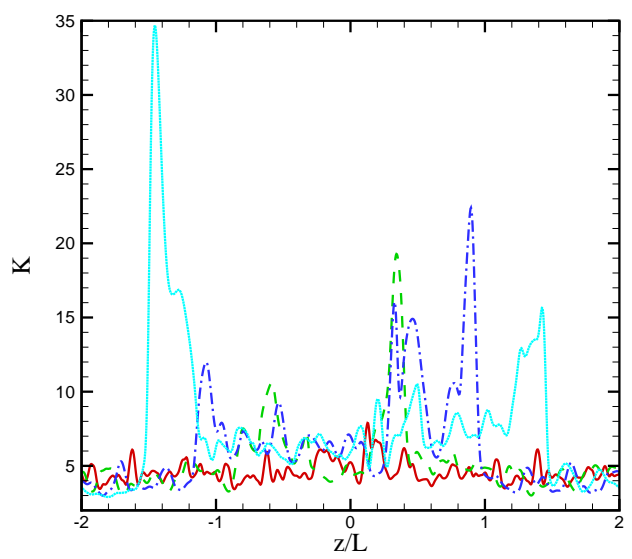

(d) $K_{\partial w / \partial y}$.

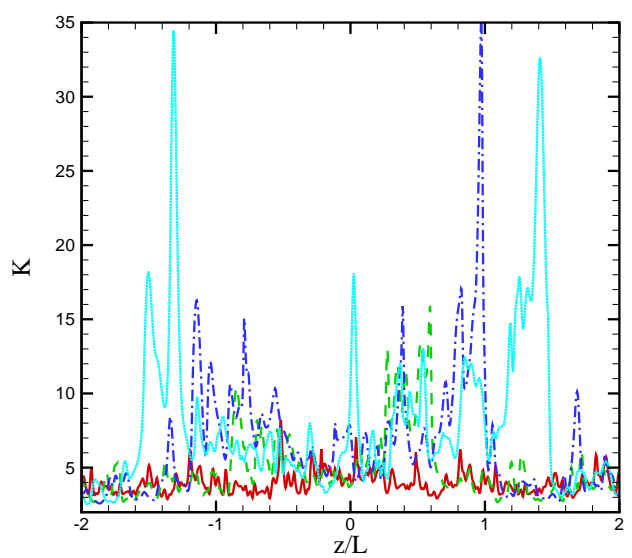

(f) $K_{\partial w / \partial z}$.

Figure 16. Spatial distribution of skewness and kurtosis at $5 \tau$ (red), $10 \tau$ (green), $15 \tau$ (blue), and $20 \tau$ (gray) at $k_{0}=4$. 


\section{Conclusions}

The evolution of a freely decaying isotropic turbulent field is investigated through DNS and important integral quantities of the flow field are reported till late times. This field is then used to perturb an interface separating two fluids in the absence and presence of a gravitational field. The current configuration is Rayleigh-Taylor unstable. The bubble and spike amplitudes are reported and a self-similar growth is observed for the simulations without gravity. Important length scales in each direction are measured to investigate the isotropy of the flow field at different scales. The flow remains isotropic at the Kolmogorov length scales where the kinetic energy is dissipated while the flow becomes anisotropic at the Taylor microscale for the simulations with gravity. Flow intermittency in different directions at small scales is measured. The RT unstable set-up shows much higher levels of intermittency. The comparison between simulations with and without gravity suggests that the initial anisotropy in the composition is not a sufficient condition to make the velocity field anisotropic and gravity is the dominant factor forcing the velocity field to become anisotropic.

\section{Acknowledgments}

The authors gratefully acknowledge discussions with Dr. Bruce Fryxell and Dr. Eric Myra. This research was supported in part by the DOE NNSA/ASC under the Predictive Science Academic Alliance Program by Grant No. DEFC52-08NA28616. This work used the Extreme Science and Engineering Discovery Environment (XSEDE), which is supported by National Science Foundation grant number OCI-1053575.

\section{References}

${ }^{1}$ Sharp, D., "An overview of Rayleigh-Taylor instability," Physica D, Vol. 12, 1984, pp. 3-18.

${ }^{2}$ Dimotakis, P., "Turbulent mixing," Annu. Rev. Fluid. Mech, Vol. 37, 2005, pp. 329-356.

${ }^{3}$ Cook, A. and Dimotakis, P., "Transition stages of Rayleigh-Taylor instability between miscible fluids," J. Fluid Mech., Vol. 443, 2001, pp. 69-99.

${ }^{4}$ Cabot, W. H. and Cook, A. W., "Reynolds number effects on Rayleigh-Taylor instability with possible implications for type-Ia supernovae," Nature Phys., Vol. 2, 2006, pp. 562-568.

${ }^{5}$ Movahed, P. and Johnsen, E., "Numerical simulations of the Richtmyer-Meshkov instability with reshock," $20^{\text {th }}$ AIAA Computational Fluid Dynamics Conference, , No. 2011-3689, 2011.

${ }^{6}$ Dimonte, G., Youngs, D. L., Dimits, A., Weber, S., Marinak, M., Wunsch, S., Garasi, C., Robinson, A., Andrews, M., Ramaprabhu, P., Calder, A., Fryxell, B., Biello, J., Dursi, L., MacNeice, P., Olson, K., Ricker, P., Rosner, R., Timmes, F., Tufo, H., Young, Y., and Zingale, M., "A comparative study of the turbulent Rayleigh-Taylor instability using high-resolution three-dimensional numerical simulations: The Alpha-Group collaboration," Phys. Fluids, Vol. 16, No. 5, 2004, pp. 1668-1693.

${ }^{7}$ Cabot, W. and Zhou, Y., "Statistical measurements of scaling and anisotropy of turbulent flows induced by RayleighTaylor instability," Phys. Fluids, Vol. 25, No. 1, JAN 2013.

${ }^{8}$ Reid, R. C., Prausnitz, J. M., and Poling, B. E., The Properties of Gases and Liquids, McGraw-Hill, New York, 4th ed., 1987.

${ }^{9}$ Movahed, P. and Johnsen, E., "A solution-adaptive method for efficient compressible multifluid simulations, with application to the Richtmyer-Meshkov instability," J. Comput. Phys., Vol. 239, 2013, pp. 166-186.

${ }^{10}$ Blaisdell, G. A., Spyropoulos, E. T., and Qin, J. H., "The effect of the formulation of nonlinear terms on aliasing errors in spectral methods," Appl. Numer. Math., Vol. 21, 1996, pp. 207-219.

${ }^{11}$ Ducros, F., Laporte, F., Souleres, T., Guinot, V., Moinat, P., and Caruelle, B., "High-order fluxes for conservative skew-symmetric-like schemes in structured meshes: Application to compressible flows," J. Comput. Phys., Vol. 161, 2000, pp. 114-139.

${ }^{12}$ Pirozzoli, S., "Numerical methods for high-speed flows," Ann. Rev. Fluid Mech., Vol. 43, 2011, pp. $163-194$.

${ }^{13}$ Gottlieb, S. and Shu, C. W., "Total variation diminishing Runge-Kutta schemes," Math. Comp., Vol. 67, 1998, pp. 73-85.

${ }^{14}$ Thompson, K., "Time-dependent boundary-conditions for hyperbolic systems," J. Comput. Phys., Vol. 68, 1987, pp. 1-24.

${ }^{15}$ Lee, S., Lele, S., and Moin, P., "Eddy shocklets in decaying compressible turbulence," Phys. Fluids, Vol. 3, 1991, pp. 657-664.

${ }^{16}$ Johnsen, E., Larsson, J., Bhagatwala, A. V., Cabot, W. H., Moin, P., Olson, B. J., Rawat, P. S., Shankar, S. K., Sjogreen, B., Yee, H. C., Zhong, X., and Lele, S. K., "Assessment of high-resolution methods for numerical simulations of compressible turbulence with shock waves," J. Comput. Phys., Vol. 229, 2010, pp. 1213-1237.

${ }^{17}$ Monin, A. S. and Yaglom, A. M., Statistical Fluid Mechanics, Vol. 2, MIT Press, Cambridge, MA, 1975.

${ }^{18}$ Sreenivasan, K. R. and Antonia, R. A., "The phenomenology of small-scale turbulence," Annu. Rev. Fluid Mech., Vol. 29, 1997, pp. 435-472.

${ }^{19}$ Movahed, P. and Johnsen, E., "The mixing region in free shearless multi-material turbulence," 2013, pp. in preparation.

${ }^{20}$ Tordella, D. and Iovieno, M., "Small-Scale Anisotropy in Turbulent Shearless Mixing," Phys. Rev. Lett., Vol. 107, No. 19, OCT 312011.

${ }^{21}$ Tordella, D., Iovieno, M., and Bailey, P., "Sufficient condition for Gaussian departure in turbulence," Phys. Rev. E, Vol. 77, No. 1, 2008. 
${ }^{22}$ Mellado, J., Sarkar, S., and Zhou, Y., "Large-eddy simulation of Rayleigh-Taylor turbulence with compressible miscible fluids," Phys. Fluids, Vol. 17, No. 7, 2005.

${ }^{23}$ Olson, B. J. and Cook, A. W., "Rayleigh-Taylor shock waves," Phys. Fluids, Vol. 19, No. 12, 2007.

${ }^{24}$ Abarzhi, S. I., Gorobets, A., and Sreenivasan, K., "Rayleigh-Taylor turbulent mixing of immiscible, miscible and stratified fluids," Phys. Fluids, Vol. 17, No. 8, 2005. 